\title{
The role of ubiquitination in nerve cell development
}

Hiroshi Kawabe* and Nils Brose*

*Max Planck Institute of Experimental Medicine, Department of Molecular Neurobiology, Hermann-Rein Straße 3, D-37075 Göttingen, Germany.

Correspondence to H.K. (e-mail: kawabe@em.mpg.de) or N.B. (e-mail: brose@em.mpg.de)

\begin{abstract}
Nerve cell development in the brain is a tightly regulated process. The generation of neurons from precursor cells, their migration to the appropriate target sites, their extensive arborisation, and their integration into functional networks through synapse formation and refinement are governed by multiple interdependent signaling cascades. The function and turnover of proteins involved in these signaling cascades, in turn, are spatially and temporally controlled by ubiquitination. Recent advances have provided first insights into the highly complex and intricate molecular pathways that regulate ubiquitination during all stages of neural development and operate in parallel with other regulatory processes such as phosphorylation or cyclic nucleotide signaling.
\end{abstract}


The human brain is estimated to contain some 100 billion neurons, each of which forms, on average, 1,000 to 10,000 synaptic contacts ${ }^{1,2}$. Despite this vast complexity, the resulting neuronal networks that control information processing in the brain are highly ordered. The controlled development of individual neurons is of crucial importance for proper network formation in the brain. Correspondingly, the key phases of nerve cell development - the proliferation of neuronal precursor cells, the generation of neurons from precursor cells, the migration of neurons to their appropriate target sites, the differentiation of neurons into extensively arborized cells, and the integration of neurons into functional networks through synapse formation and refinement ${ }^{3}$ (Figure 1) - are tightly regulated by numerous external cues and intracellular signaling processes.

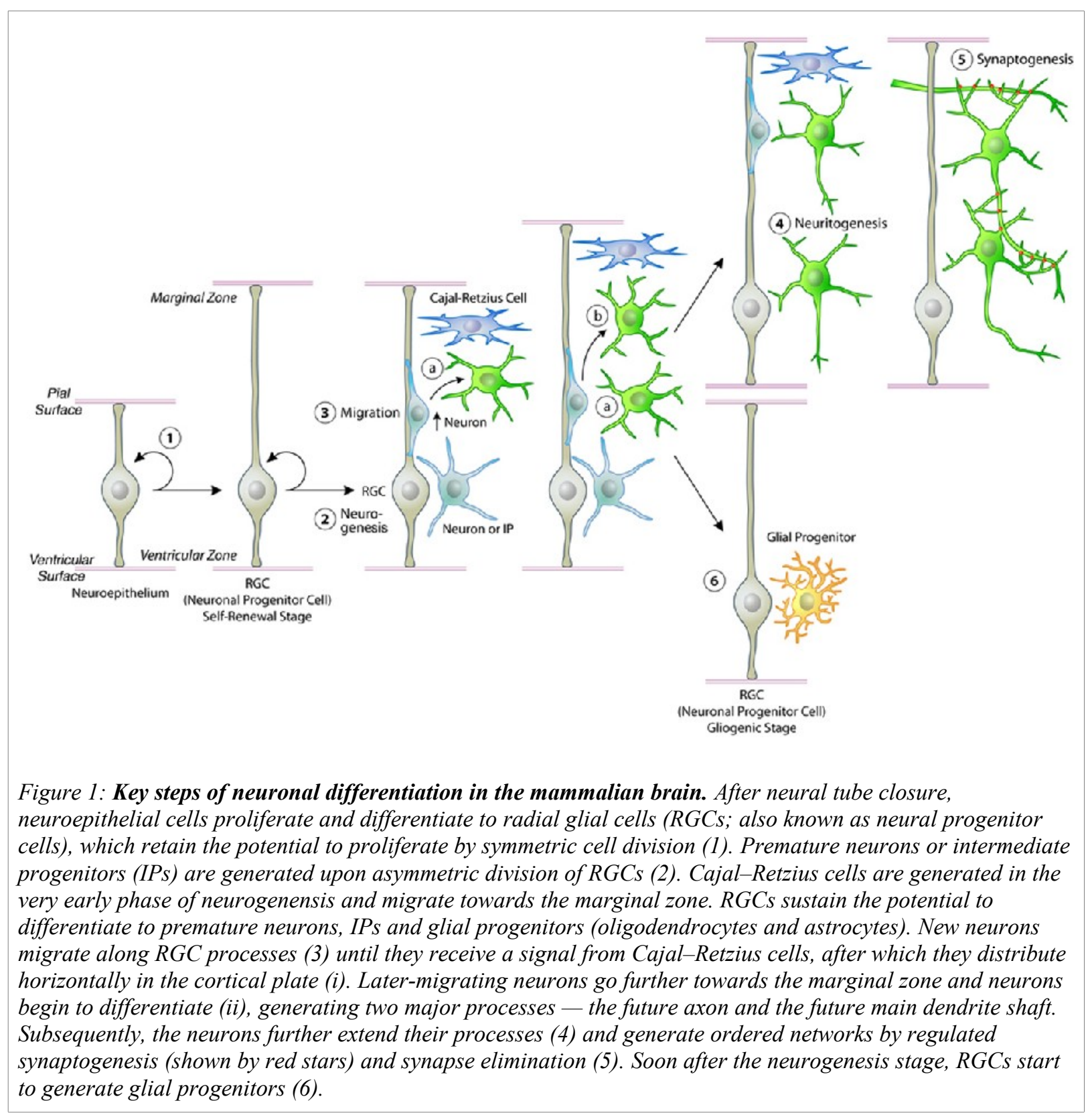

Although they mostly occur in a temporally coordinated and successive manner, the different stages of nerve cell development are partly interdependent. Dendrite development, for example, is directly influenced by synaptic inputs, and dendrite complexity affects the total number of synapses made by a given neuron ${ }^{4,5}$. Likewise, the guiding cues and signaling processes that control neuronal development are characterized by substantial crosstalk at multiple levels ${ }^{6-7,8}$. These guidance and signaling processes, in turn, are controlled by many different intracellular regulatory mechanisms. Among these, ubiquitin dependent functional modification and/or degradation of signaling proteins have recently emerged as an important and hitherto underestimated regulatory principle in nerve cell development. 
Ubiquitination is a posttranslational modification of proteins, related to phosphorylation, acylation, alkylation and other processes that modify proteins after their translation. Ubiquitination involves the conjugation of one or several 76 amino acid long ubiquitin moieties to lysine residues in substrate proteins and is catalyzed by the sequential action of three classes of enzymes (Figure 2). The specificity of ubiquitination is mainly determined by the E3 ligases, which transfer ubiquitin to substrate proteins. Some 600 different E3 ligase isoforms are encoded in the human genome ${ }^{9}$, which are classified as ReallyInteresting-New-Gene type ligases (RING finger E3 ligases) or Homologous-to-E6-AP-C-Terminus type ligases (HECT type E3 ligases). Given that the genomes of higher vertebrates encode only one or two E1 and some $30 \mathrm{E} 2$ enzymes and because E3 ligases recognize substrates via specific protein-protein interactions (Figure 2a), E3 ligases are the main determinants of the substrate specificity of ubiquitination processes. 


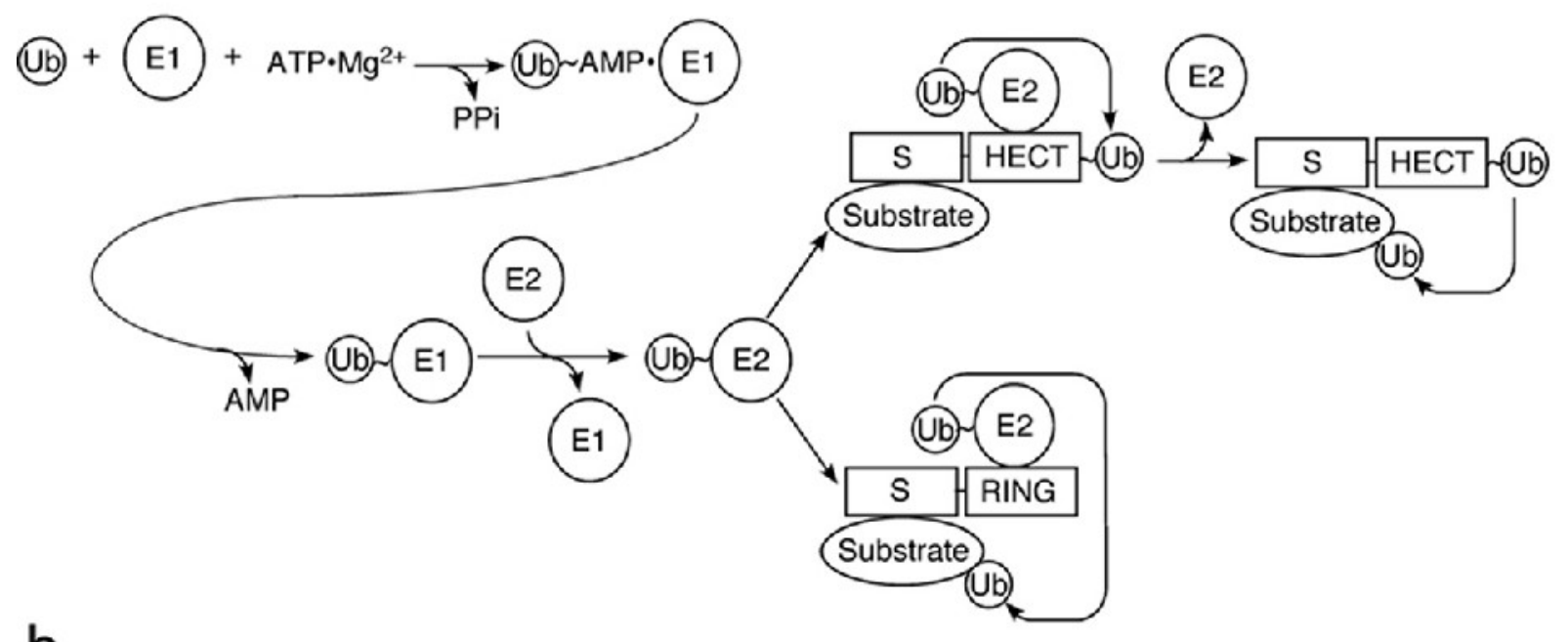

b

Polyubiqutination (K11- or K48-linked)

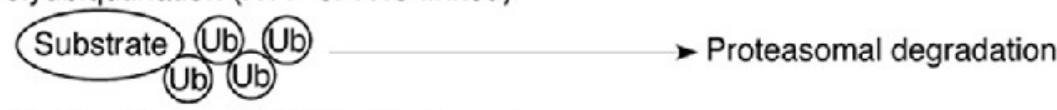

Polyubiqutination (K63-linked or linear)
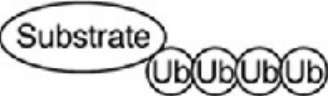

Regulation of protein functions and interactions

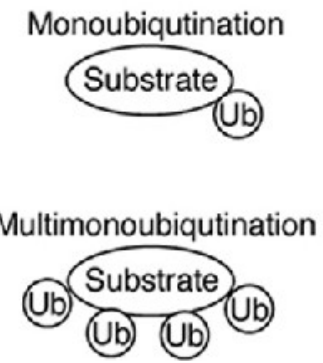

Regulation of endocytosis of transmembrane proteins, regulation of protein functions or interactions

Figure 2: The protein ubiquitylation pathway. Ubiquitylation is a sequential reaction mediated by three classes of enzymes (E1, E2 and E3). A ubiquitin-activating enzyme (E1) is conjugated with a free ubiquitin moiety through a thioester bond ( ). This reaction uses ATP $\bullet \mathrm{Mg} 2+$ to form a ubiquitin adenylate intermediate, in which ubiquitin and adenosine monophosphate (AMP) are conjugated by a high-energy thioester bond. This intermediate is first coupled to the E1 through a non-covalent bond $(\bullet)$. Ubiquitin activated in this manner is then transferred to a cysteine residue of the E1 enzyme. Active ubiquitin conjugated to the E1 enzyme through a high-energy thioester bond is subsequently transferred to a ubiquitin conjugating enzyme (E2) that, in turn, is recognized by a ubiquitin ligase (E3), of which there are two major types - homologous to E6AP carboxyl terminus (HECT)-type and RING finger-type ligases. HECT-type ligases receive the active ubiquitin from the E2 enzyme (shown by a dashed arrow), bind it covalently via a cysteine residue in the HECT domain, and subsequently transfer it to a lysine residue in the ultimate ubiquitylation substrate protein (shown by a dashed arrow), which is recognized by the substrate recognition domain of the E3 ligase (S). By contrast, the RING finger-type ligases transfer the active ubiquitin directly from the E2 enzyme to the ultimate ubiquitylation substrate protein without forming a covalent bond (shown by a dashed arrow). The human genome encodes two E1, approximately 30 E2 and about 600 E3 enzymes. $b$ | Functional consequences of protein ubiquitylation. Lys48-linked polyubiquitin chains and probably also Lys 11-linked polyubiquitin chains are directly recognized by the proteasome. Lys63-linked polyubiquitin chains and also head-to-tail-linked linear polyubiquitin chains regulate protein function. Monoubiquitylation or multi-monoubiquitylation regulate the function or endocytosis of many proteins. PPi, pyrophosphate; Ub, ubiquitin.

While all seven lysine residues of ubiquitin can be used for of ubiquitin chain formation, lysine-48-linked (K48-linked) chains have long been thought to represent the major polyubiquitin variant in eukaryotic cells. However, recent studies showed that K11-linked and K63-linked chains, whose functions are only poorly understood, are similarly abundant ${ }^{10}$. The chain type specificity solely depends on the E2 enzyme in the case of RING finger ligases, whereas protein domains C-terminal to the HECT domains are critical determinants of the ubiquitin chain types generated by HECT type ligases ${ }^{11}$. Apart from polyubiquitin chains, many 
ligases can also mono- or diubiquitinate (via K63) substrate lysine residues ${ }^{12,13}$. Initially, the focus of research on protein ubiquitination had been on proteasome-dependent degradation of polyubiquitinated cytosolic proteins. Since the 1990s, however, a flurry of studies has shown that protein ubiquitination (e.g. mono- and diubiquitination) does not necessarily control proteasomal protein degradation but rather many other cellular processes, including cell surface expression of membrane proteins, endocytosis, protein interactions, or protein function (Figure $2 b)^{12}$.

That ubiquitination and the ubiquitin proteasome system (UPS) must play a key role in brain development was first indicated by the discovery of the ubiquitin carboxy-terminal hydrolase PGP 9.5 in somata and dendrites of differentiating neurons in rat embryos ${ }^{14,15}$. In the meantime, the analysis of ubiquitination in the developing and mature brain has become a major new focus in neuroscience, not least because ubiquitination seems to play a key role in neurodegenerative disorders ${ }^{16-18}$. Thus, understanding the molecular mechanisms by which the different phases of nerve cell development and differentiation are coordinated by ubiquitination is of substantial importance for our understanding of normal brain development and function as well as of corresponding pathological perturbations.

In this review, we discuss recent progress in unraveling the mechanisms by which protein ubiquitination regulates defined signaling pathways that control nerve cell development. Admittedly, the currently available evidence is still restricted to a limited set of example pathways. However, these examples provide an exciting view of how ubiquitination-dependent regulatory processes in neurons intercalate with other, more extensively studied regulatory principles. Currently, we see only the tip of the iceberg: Ubiquitination is likely to be a general regulatory mechanism in nerve cell development, at par with phosphorylation or cyclic nucleotide signaling with regard to complexity and functional consequences.

\section{Ubiquitination in neurogenesis and gliogenesis}

Three main types of neuronal progenitors have been identified in the developing neocortex: Neuroepithelial cells, radial glial cells (RGCs), and intermediate progenitor (IP) cells. At the ventricular zone in early neurogenesis, neuroepithelial cells proliferate by symmetric cell division, and subsequently generate neurons by asymmetric division. Neuroepithelial cells generate RGCs, which expand by symmetric cell division and undergo asymmetric division at the ventricular zone, thereby producing IPs and neurons in mid gestation and glial progenitors in late gestation ${ }^{19}$. Each IP divides only once to generate two neurons in the subventricular zone, a more apical part of the developing cortex. Self-renewal of progenitor cell populations and their transition into neurogenic and gliogenic stages are controlled by a set of extracellular cues and cell intrinsic signaling pathways of five major types: WNT signaling, Notch signaling, hedgehog signaling, receptor type serine/threonine kinase signaling (e.g. via TGF- $\beta$ receptors), and signaling via receptor type tyrosine kinases (e.g. via Trk, FGF, or EGF receptors) ${ }^{20,21}$. Each of these signaling pathways is influenced by ubiquitination in one way or another. For example, it has long been known that canonical WNT signaling blocks phosphorylation of $\beta$-Catenin and its subsequent polyubiquitination and degradation by the UPS ${ }^{22},{ }^{23}$. However, the most profound and extensive recent progress has been made with regard to Notch signaling.

Ubiquitination and direct regulation of Notch signaling. Recently, the Notch pathway, whose activation inhibits neuronal differentiation, was identified as a major target of regulation by ubiquitination pathways (Figure 3). Notch signaling is triggered by the intercellular interaction between Notch ligands [i.e. Delta-Like (DLL) proteins or Jagged-1, which are induced by the proneuronal gene Neurogenin-1 (Ngn-1)] and the Notch receptor, which is expressed on the surface of RGCs. This interaction induces gamma-secretase activity, which cleaves the intramembrane domain of the Notch receptor to release the Notch intracellular domain (NICD) into the RGC cytosol. NICD then activates genes of the HES family of basic helix-loophelix (bHLH) transcriptional repressors, which, in turn, downregulate proneuronal bHLH genes [i.e. Ngn and Mash1] to maintain the developmental potential of RGCs as the neural/glial precursor cells and to prevent them from differentiation into neurons or IPs ${ }^{24}$. 


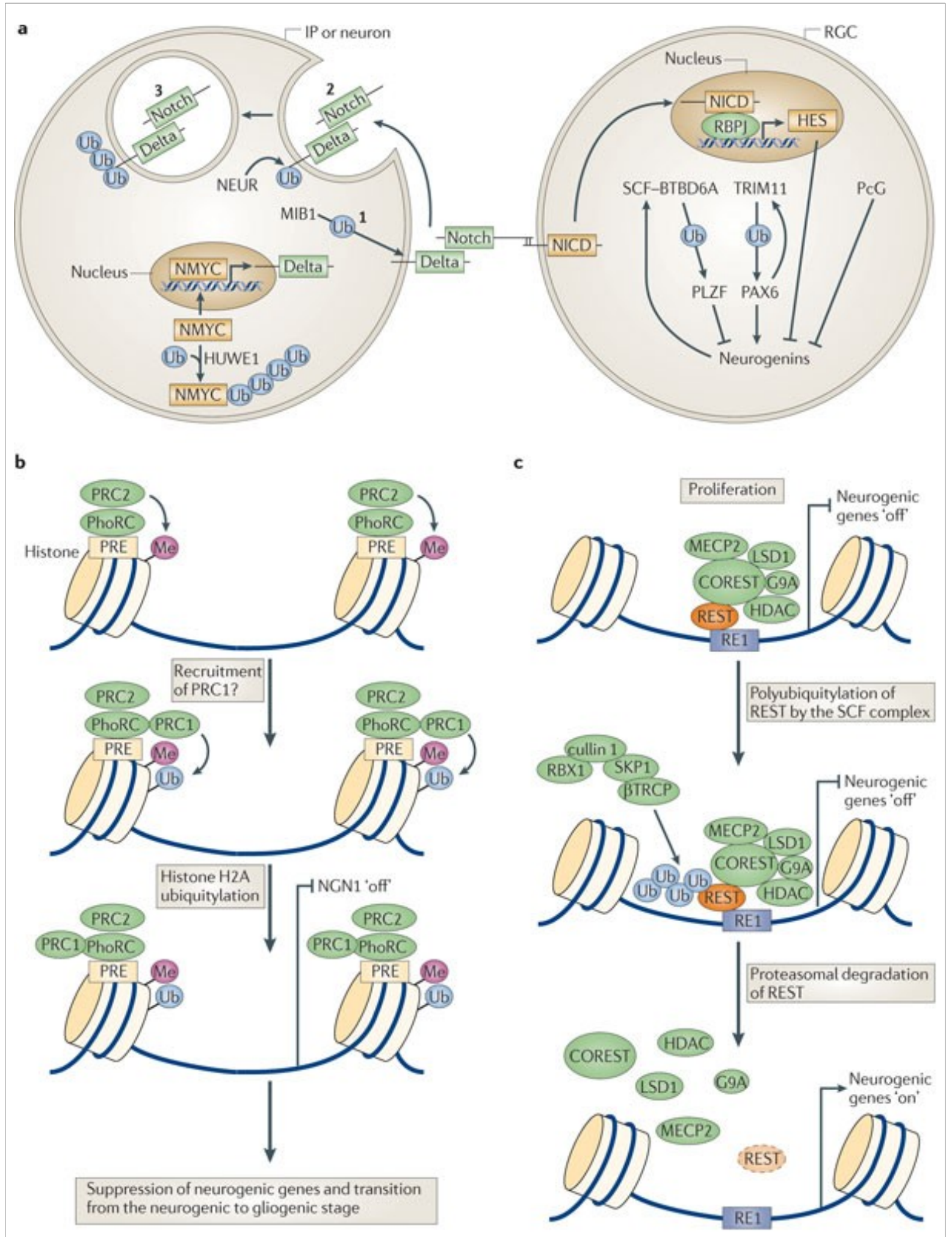

Figure 3: Figure 3 | Regulation of neurogenesis by ubiquitylation. 
Figure 3 : Regulation of neurogenesis by ubiquitylation. Lateral inhibition by the Notch pathway is regulated by multiple ubiquitylation cascades. Newly generated neurons or intermediate progenitors (IPs) (left cell) express the transmembrane Notch ligand Delta. The cytoplasmic tail of Delta is ubiquitylated by mind bomb1 (MIB1) (1), which triggers the endocytosis of the complex of Delta and the extracellular cleaved-off region of the Notch receptor (2). Subsequently, the E3 ubiquitin ligase neuralized (NEUR) ubiquitylates endocytosed Delta to drive endocytosis to late endosomes (3). This endocytosis machinery is necessary for activation of the Notch pathway in radial glia cells (RGCS). In IPs, the transcription promoter NMYC (which stimulates Delta-like 3 (DLL3) expression) is negatively regulated through polyubiquitylation by HUWE1 (also known as HECT, UBA and WWE domain-containing protein 1). This negative control of Notch activation counteracts the positive regulation by MIBI and NEUR. In RGCs, upon binding of Delta to the Notch receptor (right cell), the intracellular region of the Notch receptor is cleaved by $\gamma$-secretase. The Notch intracellular domain (NICD), together with recombining binding protein suppressor of hairless (RBPJ), promotes the transcription of the basic helix-loop-helix proteins HES1 (also known as hairy and enhancer of split 1) and/or HES5, which suppresses the proneuronal neurogenin genes. Expression of these genes is controlled by at least three independent ubiquitylation pathways: SCF (including BTB (POZ) domain-containing 6 (BTBD6)), TRIM11 (also known as tripartite motif-containing protein 11) and polycomb group (PcG). The SCF-BTBD6 complex is a macromolecular E3 ligase complex whose substrate specificity is determined by the adaptor protein BTBD6A. This SCF ligase complex reduces lateral inhibition through feedback, whereas TRIM11 and the PcG have the potential to promote lateral inhibition by suppressing the expression of neurogenins. $b \mid$ Monoubiquitylation of histone $H 2 A$ by the polycomb repressive complex 1 (PRC1) downregulates neurogenic genes (for example, neurogenin 1 (NGN1)). Phorepressive complex (PhoRC) recognizes the PC response element (PRE) sequence in the promoter region of neurogenic genes and recruits the PRC2 lysine N-methyltransferase complex (upper panel). The E3 ubiquitin-protein ligase RING2 (also known as RINGIB)-containing PRC1 E3 ligase complex is recruited to the nucleosome, where it ubiquitylates histone H2A (middle panel). Whether this recruitment process is dependent on PRC2-mediated histone methylation is not clear. Monoubiquitylation of H2A suppresses translation of neurongenic genes in the late phase of neurogenesis and thus promotes transition to gliogenesis (lower panel). c | Polyubiquitylation of RE1 (repressor element 1)-silencing transcription factor (REST; also known as neural-restrictive silencer factor (NRSF)) by the cullin 1-based SCF- $\beta T R C P$ (Fbox/WD repeat-containing protein $1 A$; also known as $\beta$-transducin repeat containing protein) complex releases suppression of neurogenic genes. The RE1 sequence in neurogenic genes (for example, neurogenic differentiation 1 (NEUROD1)) is recognized by REST, which functions as a scaffolding protein for REST corepressor (COREST) protein, histone lysine N-methyltransferase EHMT2 (G9A), lysine-specific histone demethylase $1 A$ (LSD1), histone deacetylases (HDACs), and methyl DNA binding protein methyl CpG binding protein 2 (MECP2) (upper panel). This multimolecular epigenetic gene suppression system remains stable until neurogenesis starts upon SCF- $\beta T R C P$ polyubiquitylation of REST (middle panel).

Polyubiquitylation of REST results in its subsequent degradation. This process is crucial for displacing G9A, LSD1, HDACs and MECP2 from the RE1 sequence of neurogenic genes and causes their subsequent activation. Me, methyl group; PLZF, zinc finger and BTB domain-containing protein 16; RBX1, RING-box 1; SKP1, S-phase kinase associated protein 1; Ub, ubiquitin. 
That the UPS is critically involved in Notch signaling has been known for some time. A series of fascinating, more recent studies have implicated the RING finger E3 ubqiuitin ligase Mind bomb (Mib) and the HECT E3 ligase Huwe1 in Notch function (Figure 3). The Mind bomb (mib) gene was initially characterized as a mutant showing increased neurogenesis in zebrafish ${ }^{25,26}$. Corresponding loss-of-function mutant fish show increased neurogenesis due to suppression of the Notch signaling pathway ${ }^{27}$. More specifically, cell transplantation experiments indicate that $m i b$ is required in signaling cells for efficient activation in of Notch in neighboring cells. Mouse Mib1 is strongly expressed in neurons and IPs, indicating that these cell types send Notch signals to RGCs during migration and are compromised in mib mutants ${ }^{28}$. Indeed, mice with a brain-specific deletion of Mib1 exhibit premature differentiation of RGCs to IPs and neurons ${ }^{28}$.

Overall, the effects of mib loss of function are similar in zebrafish and mouse, causing reduced Notch activity and consequent changes in somitogenesis, vasculogenesis, neurogenesis, and cardiogenesis ${ }^{24,27,29,30}$. In all model animals studied so far, Mib induces the endocytosis of Delta. In addition, other Notch ligands such as different Dll and Jagged family members are regulated by Mib, and Notch target genes are downregulated in Mib deficient mice ${ }^{29,31}$. A likely scenario is that Mib-mediated endocytosis of Delta facilitates Notch cleavage and signaling ${ }^{27}$.

Interestingly, Mib itself is regulated by components of the protein machinery that determines asymmetric cell division in the neuroepithelium. Planar orientation during mitosis at the apical surface of the neuroepithelium is dependent on the epithelial cell polarity. LGN, Inscutable (Insc), Par proteins (Par1, Par3, and Par6) and atypical PKC (aPKC) are all distributed in a polarized manner in dividing cells and involved in asymmetric cell division of neuroblasts in Drosophila. In LGN or Insc mutant mice, mitotic spindle orientation is abnormal, resulting in mislocalisation of IPs and reduced numbers of RGCs, indicating that planar orientation is critical for the maintenance of RGCs ${ }^{32}$. The protein kinase Par1, which is necessary for neuronal polarity formation, phosphorylates Mib and thus triggers its degradation by the UPS, which, in turn, downregulates Notch signaling and induces neurogenesis ${ }^{33}$.

In contrast to the RING finger E3 ligase Mib, which appears to promote Notch signaling, the HECT type E3 ligase Huwe1 was recently implicated in negative regulation of the Notch pathway, involving the transcription factor N-Myc (Figuse 3a). The Myc family of transcription factors is composed of three proteins, c-Myc, L-Myc, and N-Myc. N-Myc is expressed in the developing brain, and loss of N-Myc results in the downregulation of the Myc target gene cyclin D2, reduced brain size due to decreased mitosis rates of progenitor cells at the self-renewal stage, and precocious neuronal differentiation ${ }^{34}$. The HECT type E3 ligase Huwe1 binds and ubiquitinates N-Myc, thus targeting it for UPS-mediated degradation ${ }^{35}$. This pathway is a critical determinant of neuronal differentiation in vivo, as RNAi-mediated knock-down of Huwel results in an increase of the fraction of proliferating cells in the developing brain and blockade of neuronal differentiation. Loss of N-Myc suppresses the effects of loss of Huwe1 function, indicating that Huwe1 is a negative regulator of N-Myc ${ }^{35}$. Interestingly, the Notch ligand Dll3 is also a downstream component of the N-Myc pathway that controls proliferation and neuronal differentiation ${ }^{36}$. Thus, Huwe 1-NMyc signaling may act via two pathways, one involving cell-autonomous downregulation of cyclin D2 in RGCs and the other causing downregulation of Notch signaling through repression of D1l3 in neurons and IPs.

Given their specific and opposite actions on Notch signaling, Mib and Huwe1 may well be directly involved in determining the self-renewal properties of RGCs or IPs, e.g. by distributing differentially between daughter cells to define which RGCs proceed towards the neurogenic stage, as is for example the case for atypical protein kinase $\mathrm{C}(\mathrm{aPKC})$ and Par3. aPKC and Par3 are concentrated at the apical surface of neuroepithelial cells and RGCs. This polarized localization of aPKC and Par3 is the basis for their uneven distribution between the two daughter cells during asymmetric division as the cell division plane is rotated and the apical membrane remains in the $\mathrm{RGC}^{37}$. Assuming that Mib and Huwe1 indeed cooperate with aPKC and Par3 in cell fate determination during neurogenesis, it would be important to analyze how the two ligases are distributed subcellularly during symmetric and asymmetric cell division.

Regulation of proneural gene expression by ubiquitination-dependent feedback loops. Several ubiquitination pathways regulate Notch signaling without directly affecting Notch or its ligands (Figure 3). One recently discovered pathway of this type involves Pax6, a homeodomain-containing transcription factor involved in eye, brain, and pancreas development ${ }^{38-40}$. During mouse brain development, Pax6 is highly expressed in RGCs in the ventricular zone but not in migrating neurons ${ }^{41}$, indicating a role for Pax 6 in neurogenesis. The 
enhancer sequence of the proneuronal Ngn2 gene is directly recognized by Pax $6^{42}$ and Pax 6 loss suppresses the expression of Ngn2 in the developing retina ${ }^{38}$. Thus Pax6 is a positive upstream regulator of Ngn2 expression that promotes neurogenesis. In this manner, it counteracts Notch signaling, which suppresses Ngn expression and neurogenesis.

Interestingly, the RING finger type E3 ligase Trim11 binds to Pax $6{ }^{43}$. Trim11 interacts with and ubiquitinates Pax6 in vitro and expression of Trim11 in the mouse embryonic cortex is critical for downregulation of Pax $6{ }^{43}$. Conditional overexpression of Pax6 disturbs cell cycle progression and enhances neurogenesis and apoptosis in the mouse brain ${ }^{44}$. Similarly, the expression level of Pax6 is upregulated and apoptosis is induced upon RNA silencing of endogenous Trim $11^{43}$. Intriguingly, the mouse Trim 11 gene contains two Pax6 binding sites, and Trim11 transcription is enhanced by overexpression of Pax6 in cultured cells. Thus, a negative feedback loop prevents hyperactivation of Pax6 by Pax6-activated Trim 11 expression, Trim11-mediated ubiquitination of Pax6, and downregulation of Pax6 expression (Figure 3). This feedback loop may have an indirect positive effect on signaling downstream of Notch as reduced Pax6 levels will lead to reduced Ngn2 expression and inhibition of neurogenesis.

A second novel ubiquitination-mediated feedback pathway that indirectly affects Notch downstream signaling in a negative manner involves the Cullin3-based SCF type E3 ubiquitin ligase complex. SCF type E3 ligases are composed of a RING finger protein (Rbx1/Roc1/Hrt1), a Cullin scaffold protein, and an adaptor protein. The adaptor protein often contains a BTB (Bric-a-Brac, Tramtrack, and Broad Complex) domain and functions either as a monomer,, a heterodimer, or a heterotrimer.. By recognizing specific target proteins, BTB domain containing adaptor proteins, including BTB-Kelch, BTB-MATH, and BTB-PHR family proteins, determine the substrate specificity of ubiquitination. One BTB-PHR family member, Btbd6, is conserved in vertebrates ${ }^{45}$. In zebrafish, whose genome contains two Btbd6s, Btbd6a and Btbd6b, the corresponding proteins act as adaptors of the Cullin3-based SCF type E3 ubiquitin ligase complex and target the transcriptional repressor Plzfa. Btbd6a blocks Plzfa-mediated inhibition of neurogenesis by promoting Plzfa degradation and subsequent induction of Ngn1. Expression of Btbd6a mRNA, on the other hand, is induced by Ngn1. Thus, Ngn1 triggers a positive feedback loop that maintains Ngn1 expression through Btbd6a induction, followed by SCF-mediated Plzfa ubiquitination and degradation, and consequent release of Ngn1 suppression ${ }^{45}$ (Figure 3).

In summary, the two novel regulatory feedback loops involving Trim11 and Btbd6/SCF may play crucial roles in the dynamic regulation of Notch signaling. They likely interact with other UPS dependent pathways that regulate the expression of Ngn1 and its upstream repressors such as HES1/5, whose expression appears to oscillate in RGCs with a time course of 2-3 hours ${ }^{46-48}$. Trim11-mediated ubiquitination and downregulation of Pax6 leads to inhibition of neurogenesis, which complements Notch mediated suppression of Ngn2 and neurogenesis. In contrast, Btbd6 counteracts Notch signaling and promotes neurogenesis by mediating the SCF dependent ubiquitination and degradation of the transcriptional repressor Plzfa. This leads to upregulation of Ngn1 expression, which is under negative control by direct Notch signaling. Given that oscillation of HES1/5 expression is dependent on UPS activity and HES1/5 is a major suppressor of neurogenic genes, including Neurogenins, Tbr2, and other bHLH proteins, the identification of E3 ligases that target HES1/5 is extremely important as they would be candidate master regulators of brain morphogenesis. 


\begin{tabular}{|c|c|c|c|c|c|}
\hline Ligase & Substrate & Ubiquitin chain & Consequence & Target structure & Function \\
\hline \multicolumn{6}{|l|}{ Neurogenesis } \\
\hline BTBD6 & PLZF & PolyUb & Protein degradation and nuclear export & NPCs & Promotion of neurogenesis of NPCs \\
\hline HUWE1 & NMYC & PolyUb & Protein degradation & Neurons, IPs & Promotion of neurogenesis of NPCs \\
\hline MIB1 & Delta & Unknown & Endocytosis (early phase) & Neurons, IPs & $\begin{array}{l}\text { Suppression of neurogenesis of } \\
\text { NPCs }\end{array}$ \\
\hline neuralized & Delta & Unknown & Endocytosis (late phase) & Neurons, IPs & $\begin{array}{l}\text { Suppression of neurogenesis of } \\
\text { NPCs }\end{array}$ \\
\hline PRC1 & Histon & MonoUb & Transcriptional repression of neurogenin & NPCs & $\begin{array}{l}\text { Suppression of neurogenesis of } \\
\text { NPCs }\end{array}$ \\
\hline SCF-bTRCP & REST & PolyUb & $\begin{array}{l}\text { Protein degradation (desupression of } \\
\text { neurogenesis by REST) }\end{array}$ & NPCs & Promotion of neurogenesis of NPCs \\
\hline TRIM11 & PAX6 & PolyUb & Protein degradation & NPCs & $\begin{array}{l}\text { Suppression of neurogenesis of } \\
\text { NPCs }\end{array}$ \\
\hline \multicolumn{6}{|l|}{ Migration } \\
\hline $\begin{array}{l}\text { cullin } 5 \text { complex (SKP- } \\
\text { cullin } 5-\text { SOCS) }\end{array}$ & DAB1 & PolyUb & $\begin{array}{l}\text { Protein degradation (downregulation of } \\
\text { reelin signalling) }\end{array}$ & Migrating neurons & Stop of cell migration \\
\hline \multicolumn{6}{|l|}{ Neuritogenesis } \\
\hline CDC20-APC complex & ID1 & PolyUb & Protein degradation & Dendrites & Extension \\
\hline $\mathrm{CDH} 1$ - containing APC & ID2 & PolyUb & Protein degradation & Axons & Inhibition of extension \\
\hline CDH1-containing APC & SNO1 & PolyUb & Protein degradation & Axons & Inhibition of extension \\
\hline \multirow[t]{2}{*}{ NEDD4 } & RAP2 (GTP) & MonoUb & Functional Inhibition & Axons, dendrites & Extension \\
\hline & PTEN & MonoUb or PolyUb & $\begin{array}{l}\text { Regulation of localization and/or } \\
\text { degradation }\end{array}$ & Axons & Branching \\
\hline \multirow[t]{2}{*}{ SMURF1 } & $\mathrm{RHOA}(\mathrm{GDP})$ & PolyUb & Protein degradation & Axons, dendrites & Axon specification, neurite extension \\
\hline & PAR6 & PolyUb & Protein degradation & Axons, dendrites & Axon specification, neurite extension \\
\hline SMURF2 & RAP1B (GDP) & PolyUb & Protein degradation & Axons & Axon specification \\
\hline \multicolumn{6}{|l|}{$\begin{array}{l}\text { Synapse Formation and } \\
\text { Elimination }\end{array}$} \\
\hline PHR & DLK1 (and ALK) & PolyUb & $\begin{array}{l}\text { Protein degradation (downregulation of } \\
\text { DLK1, MKK4 and PMK3) }\end{array}$ & Neurons & Promotion of synaptogenesis \\
\hline SKR1 & Unknown & Unknown & Unknown & Neurons & Promotion of synapse elimination \\
\hline
\end{tabular}




\section{Table 1 | E3 ubiquitin ligases in neuronal development.}

ALK, anaplastic lymphoma kinase; APC, anaphase-promoting complex; Btbd6, BTB/POZ domain containing $6 ; \cdots$-TrCP, $\therefore$ H́transducin repeat-containing protein; Cdc20, cell division cycle 20; Cdh1, Cdc20 homolog 1; DLK-1, dual-leucine zipper kinase-1; Huwe1, HECT, UBA, and WWE domain containing 1; Id, inhibitor of DNA binding/differential; IP, intermediate progenitor cell; mDab1, mammalian Disabled; Mib, Mind bomb; MKK-4, MAP kinase kinase 4; Nedd4-1, neuronal precursor expressed developmentally downregulated protein 4-1; N-Myc, v-myc myelocytomatosis viral related oncogene, neuroblastoma derived; NPC, neuronal progenitor cell; Par6, partitioning defective 6; Pax6, paired box gene 6; Phr1, PAM, highwire, and RPM-1; Plzf, promyelocytic leukaemia zinc finger protein; PMK-3, P38 MAP kinase family 3; PRC1, protein regulator of cytokinesis 1; PTEN, phosphatase and tensin homolog; Rap, Ras-related protein; REST, RE1 silencing transcription factor; RhoA, Ras homolog gene family, member A; RPM-1, Regulator of presynaptic morphology-1; SCF, Skp, Cullin, F-box containing complex; SKR-1, Skp-1 related-1; Smurf, SMAD ubiquitination regulatory factor; SnoN, Ski-1-related novel protein N; SOCS, suppressor of cytokine signaling; Trim11, tripartite motif-containing 11. 
Ubiquitination-dependent epigenetic control of gene activity in neurogenesis and gliogenesis. Apart from extracellular cues and cell-intrinsic signaling cascades, epigenetic modifications play a key role in the transition from the neurogenic to the gliogenic phase in progenitor cells. Particularly interesting in this regard are recent discoveries of an intricate interplay between DNA methylation and histone modifications such as acetylation, methylation, or ubiquitination.

DNA or histone methylation of promoter regions suppresses transcription of proneuronal genes ${ }^{49}$ and glial genes ${ }^{50}$, and conversely methylation of non-promoter regions of neurogenic genes can promote the transcription of these genes ${ }^{51}$. The promoter regions of many glial genes are hypermethylated in RGCs and associated with MeCP2 prior to entering the gliogenic stage ${ }^{52,53}$, and demethylation is required for dissociation of $\mathrm{MeCP} 2{ }^{50}$, expression of the relevant genes, and astrocyte differentiation ${ }^{54}$. Indeed, the brain specific conditional deletion of DNA methyltransferase (Dnmt1) causes a dramatic increase of glial proteins and precocious astroglial differentiation ${ }^{50}$. Two E3 ubiquitin ligases have recently been implicated in the epigenetic control of neurogenesis and gliogenesis: Ring1B, which acts as a ubiquitin ligase for histone $\mathrm{H} 2 \mathrm{~A}$, and the SCF-associated protein $\beta$-TrCP ( $\beta$-transducin repeat containing protein), which targets REST/NRSF (RE1 silencing transcription factor or Neuron-Restrictive Silencing Factor).

Ring1B operates in the context of Polycomb group (PcG) complexes, which are multimeric protein complexes that repress gene expression by chemically modifying histones, either by trimethylation or by monoubiquitination (Figure 3b). There are three classes of PcG complexes, Pho-repressive complex (PhoRC), Polycomb repressive complex 1 (PRC1), and Polycomb repressive complex 2 (PRC2), of which $\mathrm{PRC} 1$ is an E3 ligase for histone $\mathrm{H} 2 \mathrm{~A}$ while PRC2 trimethylates histone H4. The three PcG complexes are thought to cooperate via a mechanism that is initiated by the recognition of a DNA element called PcG response element (PRE) by a component of the PhoRC complex, Pho. This serves as a scaffold to recruit $\mathrm{PRC} 2$, and trimethylation of histone $\mathrm{H} 4$ by PRC2 facilitates the interaction between PRC1 and the target nucleosome ${ }^{55-57}$ (Figure 3b).

The PRC1 complex contains either Ring1A or Ring1B, which are essential E3 ligases mediating the monoubiquitination of histone $\mathrm{H} 2 \mathrm{~A}$ and consequent silencing of target genes ${ }^{56,57}$. Ring1 A operates mainly in non-neuronal tissues ${ }^{57}$, whereas Ring1B regulates neuronal and glial differentiation in the developing mammalian brain ${ }^{49}$. Neuronal precursor cells lacking Ring1B show increased Ngn1 levels, indicating that $\mathrm{Ngnl}$ is a target gene of PRC1/Ring1B ${ }^{49}$. Consistently, a prolonged neurogenic phase and a delayed onset of gliogenesis in brain is seen upon Ring1B deletion in mice, and a similar change in cell fate is observed upon inactivation of the PRC2 component Ezh2, which is a histone lysine N-methyltransferase. Thus, the epigenetic modification of histone proteins at the promoter region of the Ngnl gene and other genes by the coordinated action of PhoRC, PRC2 and PRC1 seems to play a crucial role in the transition from the neurogenic to the gliogenic phase of precursor cells (Figures 1 and $3 b$ ). However, the exact sequence of events is still disputed. For instance, the PRE binding protein Pho can also directly bind PRC1, indicating that histone trimethylation at the target region by PRC2 may not be required for PRC1 recruitment ${ }^{58-60}$.

A second more recently discovered ubiquitination-sensitive pathway that controls DNA modifications during neurogenesis and gliogenesis involves REST (Figure 3c). REST or NRSF is a transcriptional repressor and contains a central zinc finger DNA binding domain that is flanked by two repressor domains. The DNA binding domain of REST recognizes the 23 base-pair repressor element 1 (RE1) within promoter regions of multiple neuron specific genes ${ }^{61,62}$ and represses these genes in non-neuronal cells. CoREST is a major binding partner of REST and, in turn, recruits histone $\mathrm{H} 3$ lysine 9 methyltransferase (G9a), histone H3 lysine 4 demethylase (LSD1), histone deacethylase (HDAC), and MeCP2 to RE1 ${ }^{63-65}$, thus forming a core platform for epigenetic modification of target genes.

Importantly, neuronal differentiation during brain development is accompanied by UPS-dependent degradation of REST in the early phase of neurogenesis ${ }^{66}$. The underlying mechanism involves K48-linked polyubiquitination of REST by the Cullin1-based SCF- $\beta$-TrCP complex composed of the RING finger domain protein Rbx1/Roc1/Hrt1, the Cullin-1 scaffolding protein, the BTB-domain containing protein Skp1, and the F-box protein $\beta$ - $\operatorname{TrCP}$, the latter of which is responsible for substrate recognition ${ }^{67}$. Accordingly, downregulation of REST correlates with upregulation of $\beta$-TrCP in differentiating neurons. In addition, RNAi-mediated knock-down of REST promotes neuronal differentiation whereas knock-down of $\beta$-TrCP has the opposite effect, and knock-down of REST is epistatic to silencing of $\beta$-TrCP, thus enhancing neuronal differentiation. Together, these findings led to the notion that the switch from the self-renewal stage 
to the neurogenic stage of precursor cells (Figure 1) is dependent upon the downregulation of REST by SCF$\beta$-TrCP (Figure 3c). Although REST also plays a role in glia cell differentiation, it is unclear if ubiquitination of REST by SCF- $\beta$-TrCP is also involved in gliogenesis ${ }^{68}$.

In summary, recent studies have discovered two novel mechanisms through which the epigenetic regulation of neurogenesis and gliogenesis is modulated by ubiquitination-dependent control pathways. The PRC1 complex containing the ubiquitin ligase Ring1B mediates monoubiquitination of histone H2A. This leads to the silencing of target genes such as Ngn 1 in precursor cells and thus promotes the transition from the neurogenic to the gliogenic phase (Figure 1). On the other hand, an SCF complex containing the F-box protein $\beta$-TrCP polyubiquitinates the transcriptional repressor REST and targets it for degradation, causing upregulation of neurogenic genes, promotion of neurogenesis, and neuronal differentiation.

The transcriptional control of neurogenic and gliogenic genes and changes in their expression profiles are of key importance during early brain development. These processes are regulated by extracellular cues such as WNT, Notch, hedgehog, or growth factors, which have long been thought to operate mainly via protein phosophorylation or protein-protein interactions. A possible involvement of protein ubiquitination as a signaling principle that can cause functional modification or degradation of target proteins has been either underestimated or ignored. The examples of Notch signaling and the control of neurogenic genes show that protein ubiquitination contributes a novel and essential signaling mechanism that synergizes with protein phosphorylation or cyclic nucelotide signaling, which usually result in a binary on/off regulation of protein function.

\section{Ubiquitination in neuronal migration}

In the developing cortex, newborn neurons migrate along radial glia cells from the ventricular or subventricular zones towards the cortical plate under the guidance of secreted cues from Cajal-Retzius cells (Figure 1). Nerve cell migration is mainly achieved by the extension of cellular protrusions in the direction of migration, followed by nucleokinesis, i.e. movement of the nucleus, in the direction of migration. These processes are mediated by the coordinated rearrangement of the cellular cytoskeleton and the cell membrane, which, in turn, is controlled by multiple cell surface receptors, cell adhesion proteins, and intracellular signaling cascades.

Ubiquitination-dependent feedback control of Reelin function. The extracellular protein Reelin, which is secreted by the Cajal-Retzius cells in the marginal zone of the developing cortex, plays a particularly important role in neuronal migration during cortical development (Figure 4). Disruption of Reelin function causes a perturbation of the layered cortical cytoarchitecture ${ }^{69}$. Secreted Reelin is recognised by the VLDL receptor (VLDLR), APOER2, or protocadherins in migrating neurons ${ }^{70-72}$. Correspondingly, simultaneous loss of APOER2 and VLDLR function causes the same phenotypic consequences that are seen after loss of Reelin function ${ }^{73}$. These findings indicate that extracellular Reelin, neuronal APOER2, and VLDLR function in the same signaling pathway to control nerve cell migration, but the mechanism by which Reelin signaling ultimately affects the neuronal cytoskeleton during cell migration are still largely unknown. 


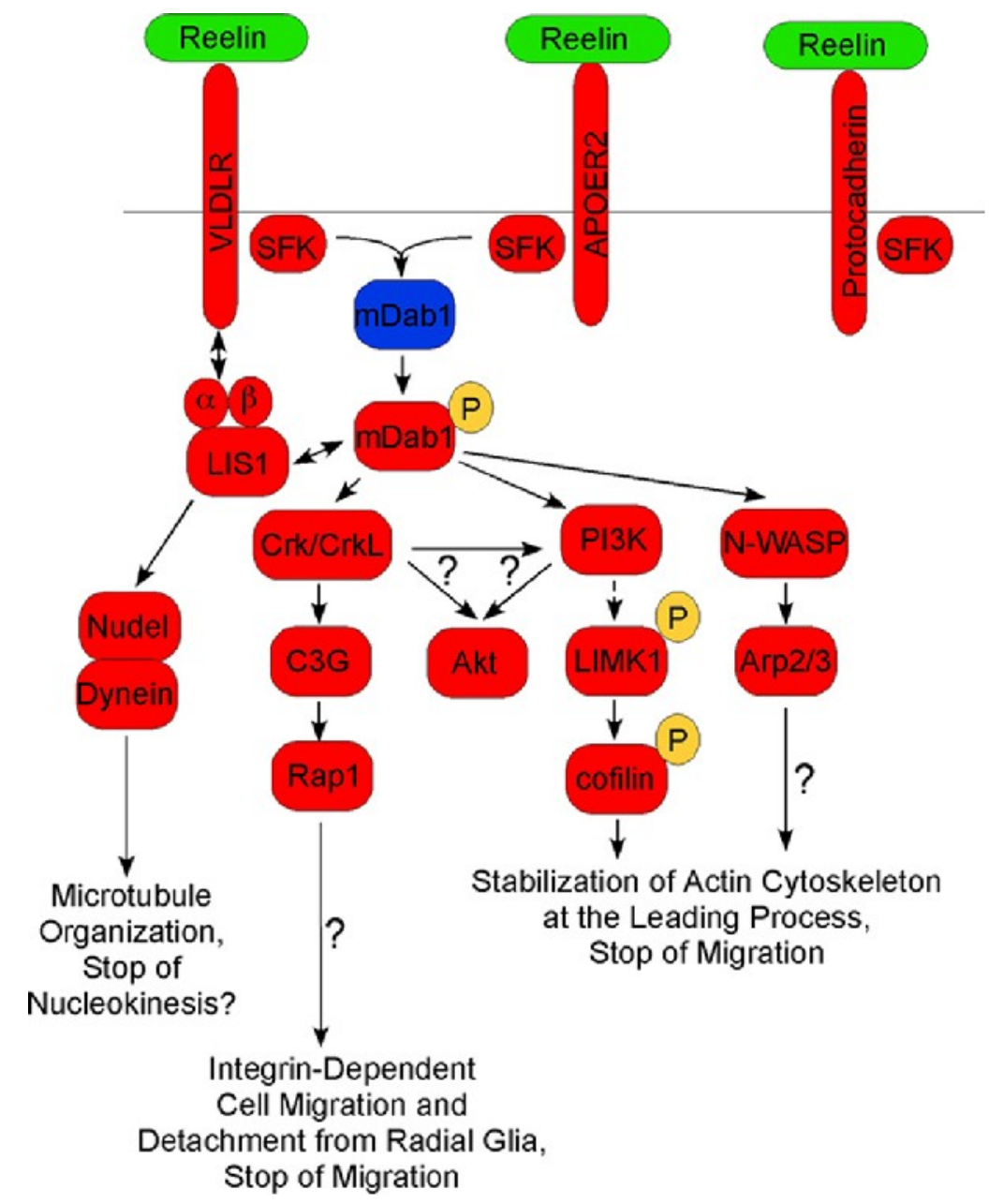

Figure 4: Molecular pathways in reelin signalling. Reelin is an extracellular protein secreted from CajalRetzius cells at the marginal zone. Reelin interacts with three transmembrane receptors expressed in migrating neurons; very low-density lipoprotein receptor (VLDLR), low-density lipoprotein receptor-related protein 8 (APOER2; also known as apolipoprotein E receptor 2) and protocadherins. These receptors associate with SRC-family kinases (SFKS), whose kinase activities are induced by reelin binding to VLDLR and APOER2 and which phosphorylate (shown by orange circles) disabled homologue 1 (DAB1).

Phosphorylated and thus active DAB1 transduces the reelin signal to all known downstream signalling cascades and is therefore of central importance in signalling the arrest of neuronal migration near the marginal zone. Lissencephaly type 1 (LIS1) forms a complex with the dynein motor complex, including nuclear distribution protein nude-like 1 (NUDEL) and cytoplasmic dynein 2 heavy chain 1 (also known as dynein heavy chain). Both LIS1 and NUDEL are crucial for neural migration as they regulate the motor function of dynein and microtubule organization. This could play a key part in the arrest of nucleokinesis in response to the reelin signal. DAB1 associates with adaptor molecule CRK and CRK-like (CRKL) in a phosphorylationdependent manner. This interaction leads to activation of RAP guanine nucleotide exchange factor $1(C 3 G)$, the guanine nucleotide exchange factor $(G E F)$ for the small GTPase RAP1, resulting in the activation of integrin. Intriguingly, phosphorylation of serine/threonine-protein kinase AKT is also positively regulated by $C R K$ and CRKL, indicating that these kinases are upstream regulators of the phosphoinositide 3-kinase (PI3K)-AKT pathway. Phosphorylation of cofilin through the PI3K-LIM domain kinase 1 (LIMK1) pathway is crucial for stabilization of the actin cytoskeleton and arrest of cell migration. This signal may be coupled with activation of neural Wiskott-Aldrich syndrome protein (NWASP) and the actin-related protein 2 (ARP2)ARP3 complex, which in turn promotes G-actin polymerization. All of these signal transduction cascades are synchronized to coordinate growth cone regulation and nucleokinesis.

The intracellular adaptor protein $\mathrm{mDab} 1$ is essential for the transduction of the Reelin signal in migrating neurons. It associates with APOER2 and VLDLR and is phosphorylated by Fyn or Src upon Reelin stimulation ${ }^{73-76}$. mDab1 expression levels are controlled by UPS-dependent protein degradation ${ }^{77}$, and Fynmediated phosphorylation of $\mathrm{mDab} 1$ is required for its ubiquitination by an ECS (Elongin $\mathrm{B} / \mathrm{C}-\mathrm{Cullin}-2 / 5$ SOCS-box protein) E3 ligase complex ${ }^{78}$. Upon RNAi knock-down of Cullin-5, mDab1 expression is 
upregulated and neurons migrate excessively, leading to a buildup of neurons at the top of the cortical plate

${ }^{78}$. This phenotype is also seen upon overexpression of a ubiquitination-deficient point mutant variant of $\mathrm{mDab} 1{ }^{79}$, indicating that Reelin function is controlled by a ubiquitination-dependent negative feedback loop in the course of which Reelin signaling via VLDLR/ApoER2 and Fyn causes mDab1 phosphorylation, consequent mDab1 ubiquitination by the ECS complex, and mDab1 degradation, which throttles Reelin signaling and thus determines the exact Reelin-dependent positioning of nerve cells in the developing brain.

The case of specific ubiquitination of $\mathrm{mDab} 1$ by the ECS complex provides one of the very first examples of protein ubiquitination processes in nerve cell migration that have been studied in vivo. However, the key experiments were performed by RNAi mediated knock-down of Cullin-5 in subpopulations of cortical neurons. The next obvious issue to be addressed would be the role of specific ubiquitination of mDab1 by the ECS complex in the lamination of cerebral cortex and in Reelin related developmental disorders ${ }^{80}$ using postmitotic neuron-specific conditional knock-out mouse lines for Cullin-5 and SOCS genes.

\section{Ubiquitination in neurite formation}

Already during migration, neurons develop protrusions, or neurites, that will utimately become axons and dendrites. Neuritogenesis is of critical importance for the formation of functional neuronal networks in the brain. It is controlled by many cell adhesion proteins and numerous short-range and long-range guiding cues that target sensor proteins at the growing end - or growth cone - of extending neurites and steer them through the developing tissue. Cell adhesion and activation of guidance-cue sensors on growing neurites trigger a vast set of intracellular signaling cascades that ultimately induce cytoskeletal rearrangements and changes in membrane flow that allow neurite growth cones to navigate through their environment.

The role of protein ubiquitination in the development of neuronal cell polarity and neuritogenesis has been studied extensively over the last decade. In this regard, recent discoveries indicate that the function of cell adhesion proteins and guidance-cue sensors as well as signaling processes that regulate cytoskeletal dynamics are particularly important targets for ubiquitination-dependent regulation.

Regulation of axonal guidance cues by ubiquitination. A particularly intriguing axon guidance problem arises in nervous systems with bilateral symmetry. To coordinately control the two body halves (e.g. for the coordinated movement of right an left limbs) many axons must cross the body midline to innervate cells on the contralateral side. The corresponding guidance cues, which are typically secreted or presented by cells in the midline, must attract axons before midline crossing and repel them after midline crossing in order to prevent axons from reentering the side of their origin. The Netrin1-deleted-in-colon-cancer (DCC) system, for example, mediates axon attraction at the midline, while axon repulsion is caused by the Netrin1-Unc5, Slit—Robo, and Sema3A—PlexA/Neuropilin-1 systems.

Inhibition of the UPS suppresses Netrin1-induced axonal growth cone collapse but has no effect on growth cone collapse induced by Sema3A. Accordingly, stimulation of growth cones by Netrin1 enhances local protein ubiquitination ${ }^{81}$. Thus, Netrin1-dependent repulsion of axons at the midline and consequent prevention of midline crossing requires UPS activity, which may involve polyubiquitination and degradation of DCC as part of the downstream cascade of this regulatory process ${ }^{82,83}$.

A very recent study implicated the ubiquitin-specific protease 33 (USP33) in Robo function ${ }^{84}$. Robo is destabilised in neurons when USP33 expression is knocked down by RNAi, and this USP33-mediated regulation of Robo is crucial for proper midline crossing of axons ${ }^{84}$. Considering that the UPS is not required for Slit-Robo signaling ${ }^{85}$, USP33-mediated deubiquitination may promote Robo signaling by preventing endocytosis and degradation, thereby maintaining the responsiveness of axons to Slit and thus avoiding aberrant midline crossing.

Local $\mathrm{Ca}^{2+}$ release from the growth cone endoplasmic reticulum through ryanodine receptors or inositol1,4,5-trisphosphate receptors is crucial for attraction of the growth cone, while $\mathrm{Ca}^{2+}$ influx through plasma membrane channels is crucial for repulsion ${ }^{86}$. These different $\mathrm{Ca}^{2+}$ signals are strictly regulated inside the growth cone, inducing local exocytosis to provide new plasma membrane at one side of the attracted growth 
cone or endocytosis to retrieve membrane from the repulsed side. It would now be of particular interest to test if polyubiquitination and subsequent degradation of proteins or UPS33-mediated deubiquitination control growth cone guidance in a locally restricted manner as well.

Poly- and monoubiquitination of small GTPases in neurite morphogenesis. The protein superfamily of small GTPases can be subdivided into at least five subgroups that can be distinguished based on their primary structures, the Ras, Rho, Rab, Sar1/Arf, and Ran subfamilies ${ }^{87}$. Although the members of this protein superfamily are involved in an extremely large and diverse set of cellular processes, they all operate by the same principles as biological switches or timers, where the GTP bound active form keeps sending a given signal until the GTP is cleaved to GDP (Figure 5a) ${ }^{87}$. Cycling of small GTPases between the GTP-bound and GDP-bound states is regulated by three types of proteins, GTPase activating proteins (GAPs), GDP/GTP exchange factors (GEFs) that promote the exchange of GDP for GTP, and GDP dissociation inhibitors (GDIs) that stabilise the GDP-bound state. 
a

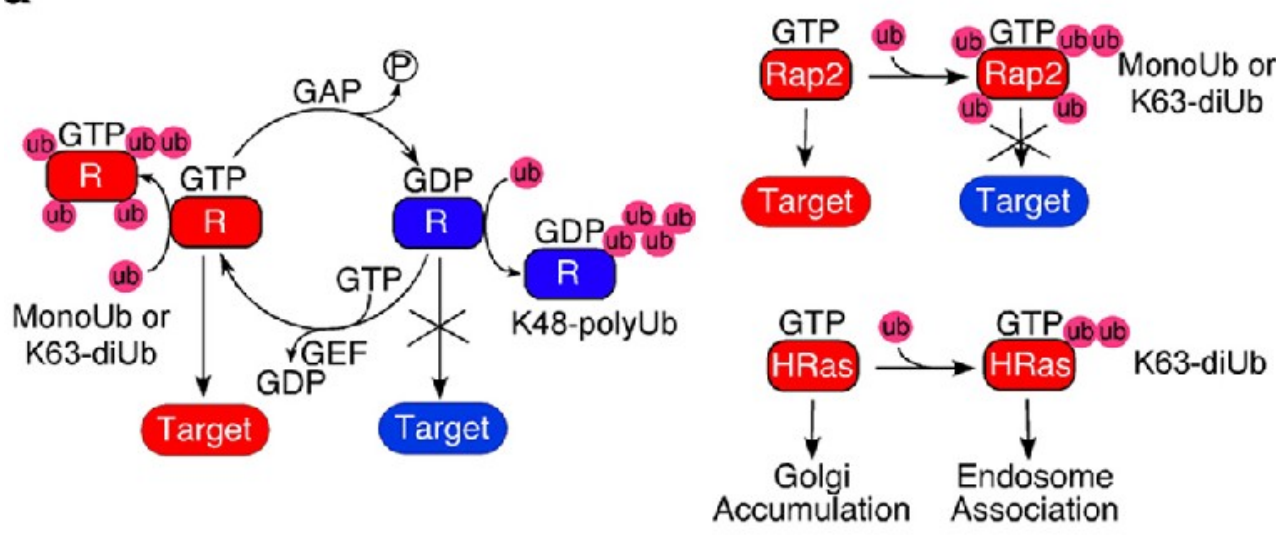

b
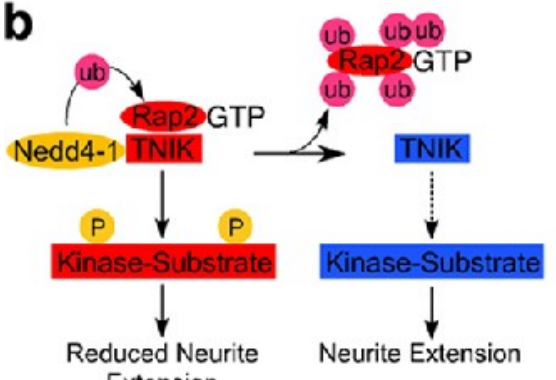

C

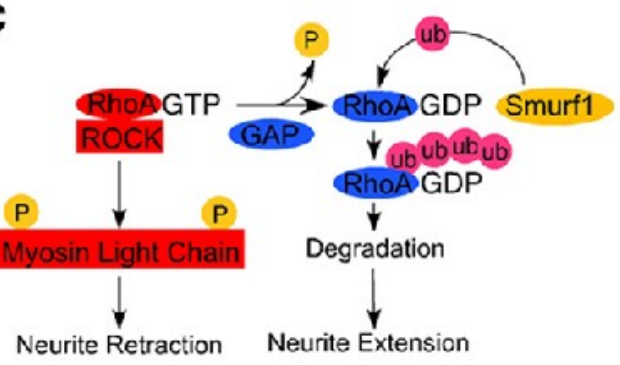

d

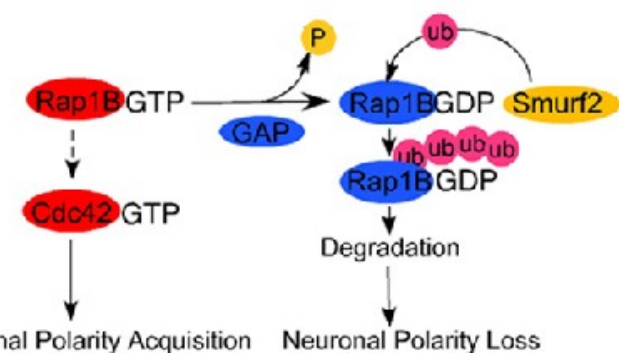

Figure 5: Regulation of neuritogenesis by ubiquitylation.. a | Several small GTPases are key ubiquitylation substrates in pathways that regulate neuritogenesis (left panel). The GTP-bound form of an active small GTPase (R, shown in yellow) interacts with, and signals towards, downstream target proteins ('Target' shown in yellow) - for example, kinases, structural proteins or guanine nucleotide exchange factors (GEFs) for other GTPases. It is inactivated by GTPase-activating proteins (GAPS) that accelerate the GTP hydrolysis activity of the GTPase. The inactive GDP-bound GTPase (R, shown in purple) has low affinity for target proteins ('Target' shown in purple) and thus signalling to downstream target proteins is terminated. GEFs reactivate the GTPases. Some active GTPases are conjugated to monoubiquitin (MonoUb) or Lys63-linked diubiquitin (diUb) - for example, HRAS and RAP2-leading to functional inactivation by interfering with the interaction with target proteins (RAP2) (middle panel) or by regulating the subcellular compartmentalization of the GTPase (HRAS) (right panel). Some inactive GDP-bound GTPases are conjugated with polyubiquitin (polyUb) chains, causing their degradation by the ubiquitin proteasome system (UPS). $b \mid$ Model of the functions of neural precursor cell expressed, developmentally downregulated 4 (NEDD4) in neurite development. NEDD4 monoubiquitylates the active GTP-bound form of RAP2, resulting in the inhibition of the RAP2 interaction with its downstream effector TRAF2 and NCK-interacting protein kinase (TNIK). Active RAP2-TNIK complexes retard neurite growth through as-yet-unknown TNIK substrates, whereas inactivation of this pathway by NEDD4-mediated RAP2 ubiquitylation results in neurite growth. $c \mid$ Model of the functions of SMURF1 in neurite development. GTP-bound RHOA activates a member of the RHO-associated protein kinase (also known as RHO-kinase (ROCK)) family, not shown, which in turn phosphorylates myosin light chain. This pathway is important for neurite retraction in response to repulsive guidance signals (for example, ephrins, SLIT3 or plexin). Once RHOA is inactivated by its GAP, it is polyubiquitylated by SMURF1. GTPases polyubiquitiylated by SMURF1 (such as RAS-related protein $1 B(R A P 1 B)$ ) are targeted for proteasomal degradation. Reduction of the total amount of RHOA in the developing neuron results in neurite extension. $d \mid$ Model of the functions of SMURF2 in neurite development. Active RAP1B is enriched at the tip of the polarized neurite, where it activates another RHO-family small GTPase, cell division cycle 42 (CDC42). This pathway is not mediated by direct interaction between the two GTPases (shown by a dashed arrow) but may involve the recruitment of a GEF for CDC42. Inactive RAP1B is recognized and polyubiquitylated by SMURF2. GTPases polyubiquitylated by SMURF 2 are targeted for proteasomal degradation. Downregulation of RAPIB protein levels upon overexpression of SMURF2 results in disrupted neuronal differentiation and loss of neuronal polarity (for example, failure to generate an axon). Thus, the SMURF-dependent pathways negatively regulate signalling by controlling the expression of small GTPases, whereas NEDD4-mediated RAP2 inhibition controls the function of this small GTPase, resulting in neurite extension. 
Members of the Rho and Ras subfamilies of small GTPases play crucial roles in neuritogenesis ${ }^{88}{ }^{89}$. RhoA promotes neurite retraction by inducing stress fiber formation, and the closely related Cdc 42 is crucial for axon development by regulating cofillin ${ }^{89,}{ }^{90}$. The Ras-subfamily GTPase Rap1B determines which of the initially formed neurites of a developing nerve cells becomes the axon by recruiting Cdc42 to the axonspecified neurite ${ }^{91}$; Rap2 promotes the retraction of the other neurites ${ }^{92}$. Several recent studies demonstrated that direct ubiquitination of Rho and Ras subfamily small GTPases by HECT-type and RING finger-type E3 ligases regulates their expression level ${ }^{93-95}$, subcellular compartmentalization ${ }^{13,96}$, or function ${ }^{97}$ (Figure 5).

The inactive GDP-bound forms of RhoA and Rap1B are polyubiquitinated by Smurf1 and Smurf2, respectively ${ }^{94,95}$, which results in the degradation of the inactive forms and downregulation of the total expression levels of these GTPases. In order to be recognized by Smurfs, the active forms of RhoA and Rap1B need to hydrolyse GTP, thus shifting the balance from the GTP bound to the GDP bound forms. Consequently, the regulation of RhoA and Rap1B by Smurf-mediated ubiquitination and degradation is dependent on the activities of GAPs. In contrast to RhoA and Rap1B, which are targeted by Smurfs when in the GDP bound state, the active GTP-bound form of Rap2 is conjugated with a single ubiquitin (monoubiquitination) or a K63-linked diubiquitin moiety (diubiquitination) by Nedd4-1 ${ }^{97}$. Rap2 ubiquitination by this mechanisms does not affect protein degradation but rather blocks the interactions of Rap2 with target proteins. A major target protein whose interaction with Rap2 is blocked by Nedd4-1mediated Rap2 ubiquitination is the kinase TNIK, which is usually activated by Rap2 binding and which promotes neurite retraction. Accordingly, loss of Nedd4-1 leads to reduced dendrite growth, which is mimicked by the overexpression of dominant active mutants of Rap2 and rescued by the overexpression of dominant inactive mutants of Rap2 or TNIK. Surpisingly - and unlike mammalian Nedd4-1 - Xenopus laevis Nedd4 appears to control axon branching rather than dendrite growth. Perturbation of Nedd 4 function in the frog by specific morpholinos or overexpression of a dominant negative Nedd4 mutant inhibits axonal branching by targeting PTEN for UPS dependent degradation ${ }^{9}$. Whether this as a general mode of Nedd4 action is currently not clear. While several studies indicated that Nedd4-1 may act as a ubiquitin ligase for PTEN ${ }^{99,10}$, deletion of Nedd4-1 in mice does not affect PTEN expression, localization, or function ${ }^{10}$, indicating that mammalian PTEN is not controlled by Nedd4-1 in vivo.

Smurf1, Smurf2, and Nedd4-1 belong to the same subfamily of HECT-type E3 ligases containing a $\mathrm{Ca}^{2+}$ binding $\mathrm{C}_{2}$ domain, two to four WW domains, and a catalytic HECT domain ${ }^{10}$. They may therefore be targets of similar activation mechanisms, such as $\mathrm{Ca}^{2+}$-dependent membrane binding, and could complement each other during nerve cell polarization and neuritogenesis. Apart from interfacing with $\mathrm{Ca}^{2+}$-signaling, crosstalk with phosphorylation is another mechanism by which HECT type E3 ligases can be regulated ${ }^{10}$. Neuronal Smurf1 is phosphorylated in a Neurotrophin (e.g. BDNF) and PKA dependent manner, which increases the ubiquitination level of RhoA. Phosphorylated Smurfl is enriched at the axonal tip and accelerates proteasomal degradation of RhoA locally. This locally restricted RhoA polyubiquitination is essential for axon acquisition.

In general, the activity of small GTPases is thought to be mainly controlled by GAPs and GEFs (Figure 5). The examples of Rap1, Rap2, and RhoA demonstrate that ubiquitination exerts an equally important regulatory influence on small GTPase function with profound consequences for neuronal polarization and neurite development. It will be important to test in future studies how extracellular guidance cues coordinate the activities of GAPs, GEFs, and E3 ligases to direct neurite growth, and to examine which other small GTPases are subject to ubiquitination dependent control as well.

Anaphase Promoting Complexes (APCS) in neurite growth. APCs are evolutionarily conserved multimeric RING finger type E3 ligase complexes. They are composed of at least thirteen proteins and utilize the WD40-domain containing proteins Cdh1 or Cdc20 as adaptors to recognize substrates ${ }^{104}$. Depending on the adaptor protein that a given APC complex utilises, the APC core has the potential to ubiquitinate multiple different substrate proteins.

APC was originally characterized as a key regulator of the cell cycle, in the course of which it targets mitotic cyclins for degradation. However, APC is also strongly expressed in postmitotic neurons. In postmitotic cerebellar granule neurons, Cdh1-APC is localized to the nucleus where it ubiquitinates the transcriptional repressor SnoN ${ }^{105,106}$. SnoN, in turn, is expressed in the internal granule layer of the cerebellum between postnatal days 6 and 13, i.e. the developmental stage during which axon growth takes place. Indeed, RNAi- 
mediated knock-down of SnoN inhibits axon growth while SnoN overexpression has the opposite effect ${ }^{106}$. Moreover, the effect of RNAi-mediated knock-down of Cdh1 is very similar to that of SnoN overexpression and rescued by simultaneous SnoN knock-down, supporting the notion that Cdh1-APC functions as a negative regulator of the transcriptional repressor SnoN by promoting its degradation. This, in turn, causes upregulation of as yet unknown genes that encode proteins with an inhibitory role in axon growth.

Compared to Cdh1-APC, the role of Cdc20-APC in nerve cell development is strikingly different ${ }^{107}$ as it controls dendrite but not axon growth. RNAi-mediated knock-down of Cdc20 results in impaired dendrite development. The relevant Cdc20-APC substrate in this process seems to be the helix-loop-helix protein Id1 (Inhibitor of DNA binding 1). RNAi-mediated knock-down of Id1 promotes dendrite growth and counteracts the effect of Cdc20 knock-down. Given that Cdc20-APC, Id1, and HDAC6, a regulator of Cdc20-APC, are all enriched at the centrosome, these findings indicate that Id 1 signaling inhibits dendrite growth whereas its Cdc20-APC dependent degradation promotes dendrite morphogenesis. However, the exact mechanism by which Id1 exerts its effect on dendrite growth is unknown.

In view of the functional differences between Cdh1-APC and Cdc20-APC, it is likely that the two APC adaptors Cdh1 and Cdc20 are differentially compartmentalised in developing neurons in order to exert their differential role. In this regard, it is important to note that Cdh1 is phosphorylated by cyclin dependent kinases $(\mathrm{Cdks}){ }^{108}$. Phosphorylation of Cdh1 blocks its nuclear import in non-neuronal cells ${ }^{109}$ and prevents the axonal morphogenesis effects of $\mathrm{Cdh} 1$ in developing neurons ${ }^{108}$, indicating that the localized ubiquitination activity of Cdh1-APC is crucial for its function in neurons.

Initially, the fact that postmitotic neurons maintain the activity and regulation APC, whose main function is in cell cycle control, came as a surprise. In the meantime, it is clear that this E3 ligase complex has multiple functions in postmitotic neurons, including dendrite and axon arborization, as described above. In addition, Cdc20-APC indirectly controls the expression of the presynaptic regulatory protein Complexin-2 by triggering the degradation of the transcription factor NeuroD2, thereby affecting late phases of presynaptic differentiation and synapse function ${ }^{110}$. Considering the diverse roles of APCs in neurons, it will be important to study how substrate recognition of this huge E3 ligase complex is regulated during neuronal circuit formation to temporally and spatially coordinate its many functions.

\section{Ubiquitination in synaptogenesis and synapse elimination}

Following neuritogenesis, brain development culminates in synaptogenesis, by which functional neuronal networks are generated (Figure 6a). The initial specificity of connections between axons and their target cells is thought to be regulated by the same type of mechanisms that are also involved in neurite guidance processes, i.e. by contact attraction (e.g. by cell adhesion proteins), contact repulsion, and attractive or repulsive morphogenic gradients ${ }^{111}$. After nascent synaptic contacts are established, they mature into fully functional synapses. 

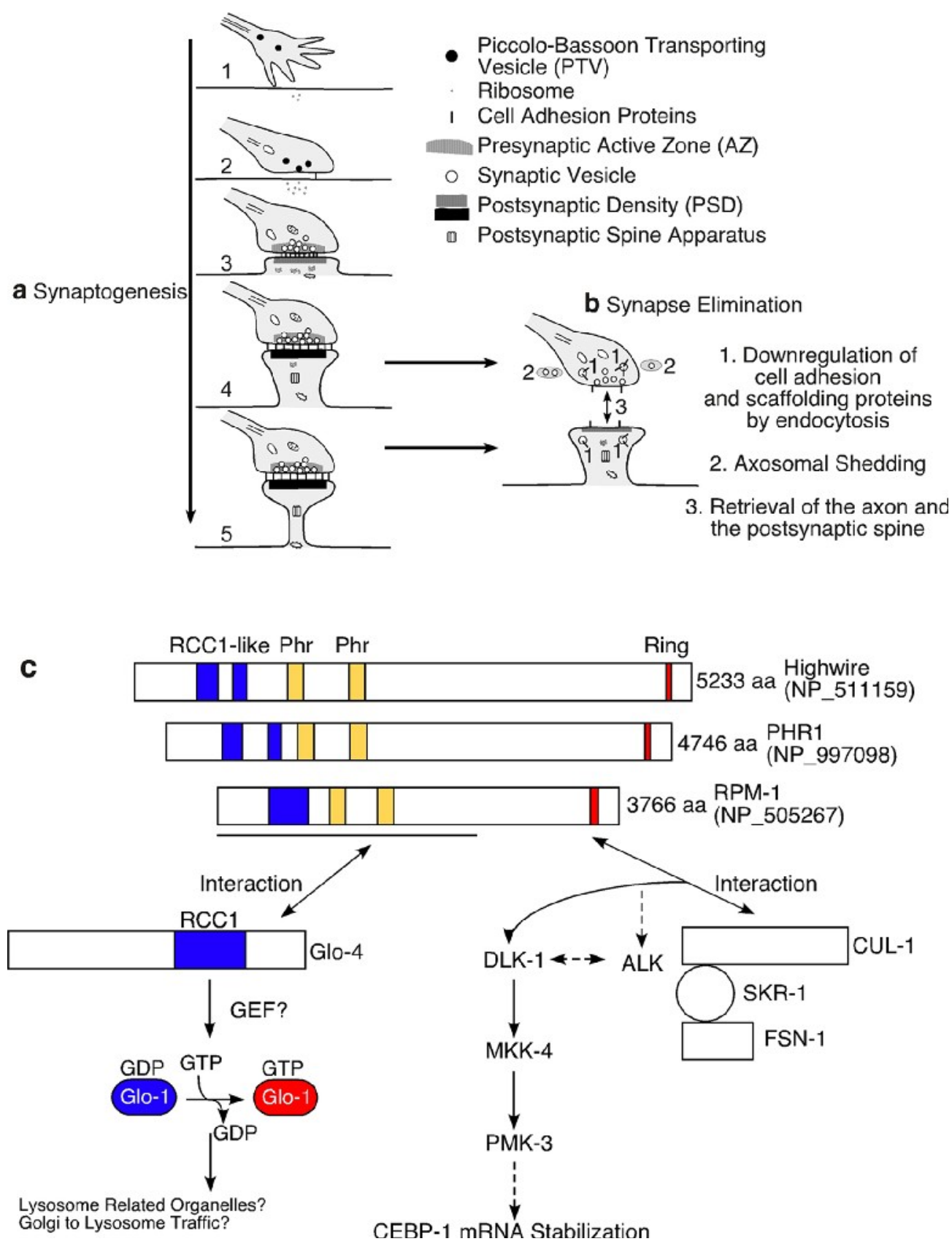

Figure 6: Regulation of synaptogenesis by PHR family ligases. a Formation and maturation of synapses. In the initial phase of synaptogenesis, the axon growth cone approaches and contacts the target cell membrane (1,2), probably driven by cell adhesion proteins (for example, cadherins, nectins or cell adhesion molecules (CADMs); shown by the vertical blue bars). Components of the presynaptic active zone are transported on piccolo-bassoon transport vesicles (PTV) (1,2; shown by filled purple circles) and deposited at the presynaptic membrane upon fusion of PTVs with the plasma membrane (presynaptic active zone is shown by the grey shaded areas in the presynaptic terminal). Subsequently, synaptic vesicles accumulate at the presynaptic terminal, and components of the postsynaptic density (PSD) accumulate at the postsynaptic site (3-5, shown by the grey shaded areas at the postsynaptic membrane). Polyribosomes that are present in the postsynapse indicate local protein synthesis $(3,4)$. Maturation of the postsynapse involves the recruitment of transmitter receptors and the spine apparatus as well as the elongation of the spine neck (4,5). b Synapse elimination can occur during and after synapse maturation. The underlying cellular processes are still largely unknown, but probably include the removal of cell adhesion and scaffolding proteins, the removal of presynaptic components by shedding of axosomes, which are then cleared by glia cells, and axon and spine retrieval. $c \mid$ Domain structures of PHR family ligases and downstream effectors. Drosophila melanogaster highwire, mouse PHR1 and Caenorhabditis elegans RPM-1 are highly homologous in their RCC1-like and PHR domains as well as in their RING finger motifs. Their amino termini, including the RCC1-like and PHR domains, interact with gut granule loss 4 (GLO-4), a putative guanine nucleotide exchange factor (GEF) for the RAB-subfamily small GTPase GLO-1. The GLO-4-GLO-1 pathway is crucial for lateendosome function, which may regulate the turnover of certain transmembrane receptors that are crucial for signal transduction in synaptogenesis. The SCF-like complex composed of RPM-1, cullin 1 (CUL-1), SKR-1 and F-box/SPRY domain-containing protein 1 (FSN-1) regulates the expression of delta homologue 1 (DLK-1) and, indirectly (shown by a dashed arrow), ALK tyrosine kinase receptor (also known as anaplastic lymphoma kinase). DLK-1 transduces signals to stabilize the $m R N A$ of the basic leucine zipper translational regulator protein CEBP-1 (CCAAT/enhancer binding protein 1). The two pathways mediated by GLO-4 and by the SCF-like complex function in parallel, to regulate synaptogenesis in C. elegans. MKK4, mitogen-activated protein kinase (MAPK) kinase 4. 
This highly coordinated maturation process, during which hundreds of specific proteins are sorted to the preand postsynaptic compartments, is controlled by synapse organizing signals such as cell adhesion proteins or transsynaptic signaling processes ${ }^{111}$. In the mature synapse, a presynaptic transmitter release site or active zone (AZ) is exactly apposed to a postsynaptic signal-receiving compartment, the postsynaptic density (PSD). AZs and PSDs contain distinct sets of adhesion and scaffolding proteins that are required for the proper equipment of the synapse with presynaptic components of the transmitter release machinery, postsynaptic transmitter receptors and signaling proteins. Given that most synapses in the mammalian forebrain are generated after birth, the activity in the developing network also has a strong influence on synaptogenesis.

In many organisms, and particularly in vertebrates, synaptogenesis is paralleled and followed by a process of synapse elimination (Figure 6b), which is of crucial importance for the refinement and specification of synaptic connectivity. In the mammalian brain, for example, up to $50 \%$ of all initially generated synapses are eliminated in late brain development ${ }^{112,113}$. Like synaptogenesis, synapse elimination is crucially dependent upon synaptic activity. With regard to vertebrate brain development, the mechanisms and molecular pathways that mediate synapse elimination are still rather enigmatic. Known pathways involve the activity of the glia-derived complement system (e.g. in the development of the visual system) and semaphorindependent synapse pruning (e.g. in hippocampus development) ${ }^{111}$.

Considering the massive protein transport and turnover processes involved, it is expected that protein ubiquitination must play a key role in the establishment of synaptic networks. However, corresponding evidence is scarce, particularly with regard to vertebrate brain development. In invertebrates, on the other hand, several recent discoveries have shed light on the role of protein ubiquitination in synaptogenesis and synapse elimination.

Ubiquitination and synapse formation in invertebrates. That protein ubiquitination is involved in the regulation of synaptogenesis was first discovered in studies on the Drosophila highwire (hiw) mutant ${ }^{114}$, which was named after the walking defects caused by the mutation ${ }^{115}$. Highwire and its mouse (Phr1) and $C$. elegans (RPM-1) orthologues form the Phr1/Highwire/RPM-1 (PHR) protein family whose members share a common domain structure with a GEF domain (RCC1-like) and a RING finger E3 ubiquitin ligase domain (Figure 6c).

Highwire is localized to the presynaptic periactive zone like several other proteins involved in synapse formation and function ${ }^{115}$, and the loss of Highwire leads to aberrant morphology of presynaptic boutons and reduced synaptic transmission ${ }^{115}$. Interestingly, the aberrant phenotype of hiw mutants is partially restored by additional loss-of-function alleles of a deubiquitinating protease, faf. This genetic interaction and the fact that Highwire contains a RING finger E3 ligase domain support the notion that a Highwire-mediated specific ubiquitination pathway regulates synaptogenesis in the fruit fly ${ }^{114}$.

The Highwire orthologue in C. elegans, RPM-1, has a related function in synaptogenesis ${ }^{16,}$,117. Loss of RPM1 causes a perturbed subcellular organisation of AZs with fewer docked vesicles. In addition, the distribution of synaptic terminals at neuro-muscular junctions is disturbed. Several independent molecular biological and genetic studies identified binding partners and downstream targets of Highwire/RPM-1 ${ }^{118-122}$.

Among these, the MAP kinases DLK-1 and MKK4 are of particular interest as they function in a linear signaling pathway that is upregulated in $r p m-1$ mutants ${ }^{119}$. DLK-1 is a MAPKKK that is strongly expressed in the $C$. elegans nervous system and can be ubiquitinated by RPM-1 in vitro ${ }^{119}$. This finding and the fact that the loss-of-function phenotype of rpm-1 mutants can be rescued at least partially by simultaneous inactivation of $d l k-1, m k k-4$, or $p m k-3$ support the notion that DLK-1 is a direct target of RPM-1. The downstream effects of RPM-1-controlled signaling via the DLK-1/MKK-4/PMK-3 pathway are still largely unknown. One possibility is that the DLK-1/MKK-4/PMK-3 pathway activates the kinase MAK-2 and the basic-leucine-zipper transcriptional regulator protein CEBP-1 (CCAAT/enhancer-binding protein-1) to regulate synaptogenesis ${ }^{123}$.

Interestingly, the F-box protein FSN-1 was recently shown to be functionally associated with RPM-1 in $C$. elegans ${ }^{124}$. Like rpm-1 mutants, $f_{s n}-1$ mutants show a significant perturbation of synaptogenesis with an uneven distribution of synaptic puncta and overgrowth of single synapses. The effect of rpm-1 loss of function is not enhanced by additional mutation of $f s n-1$, indicating that the two genes may operate in the 
same pathway. Indeed, FSN-1 forms an SCF-like complex with RPM-1, the Skp1 protein SKR-1 and the Cullin protein CUL-1, which regulates the expression level of anaplastic lymphoma kinase (ALK). This regulatory pathway appears to be directly involved in the synaptogenesis function of FSN-1 and RPM-1.

Recent biochemical and genetic studies showed that the Drosophila orthologues of FSN-1 and RPM-1, DFsn and Highwire, also interact functionally ${ }^{125}$. For example, loss-of-function mutations in $D F s n$ aggravate hypomorphic hiw phenotypes but not the effects of total loss of hiw function. Further, Drosophila DLK is upregulated in hiw or DFsn mutants, and the phenotypes of both hiw and DFsn mutants are rescued by inactivating DLK. Taken together, these finding are in nice accord with data obtained in C. elegans, further supporting the notion that Highwire/RPM-1 and FSN operate in the same pathway with DLK as a major target ${ }^{122,125}$. Even in mammals, the Highwire/RPM-1 and FSN orthologues, Phr1 and Fbxo45, seem to function in a complex at synapses ${ }^{126,127}$, but this complex may have neuronal targets other than DLK ${ }^{128}$. In addition to its role in the regulation of protein expression via its C-terminal RING finger motif, C. elegans RPM-1 interacts with the Rab GEF GLO-4 (Gut granule loss-4) through its N-terminus ${ }^{121}$. RPM-1 and GLO4 are colocalised in presynaptic terminals. Indeed, RPM-1 appears to regulate synaptogenesis through two pathways, one of which operates via $f s n-1$ and $d l k-1$ and the other via glo- 4 (Figure $6 \mathrm{c}$ ). This notion is supported by genetic experiments showing that the phenotypes of single $f_{s n-1}$ or glo- 4 loss-of-function mutations are less severe than the effects of a loss of $r p m-1$ function, while $f s n-1 ; g l o-4$ double mutants almost perfectly copy the phenotype of rpm- 1 mutants. Moreover, the phenotype of rpm- 1 loss-of-function mutants is only partially rescued by the parallel loss of $d l k-1$, and the remaining phenotypic alterations are similar to those seen in glo-4 mutants.

In summary, PHR family proteins play a key role in synapse formation, at least in invertebrates. They are negatively controlled by deubiquitiating enzymes such as Faf and they operate in an SCF-like complex with F-box proteins such as FSN-1. Known substrates are the MAPKKK DLK-1 and the anaplastic lymphoma kinase ALK, but it is currently still unkown how exactly DLK-1 or ALK regulation by PHR family proteins influences synaptogenesis.

Ubiquitination and synapse elimination in invertebrates. During C. elegans development, axons of hermaphrodite-specific egg-laying motor neuron (HSNs) initially form supernumerous synapses with the vulval muscle, many of which are eliminated later in development. This synapse elimination process is crucially regulated by the synaptic adhesion molecule SYG-1, with synapses that contain SYG-1 being spared from elimination while SYG-1 deficient synapses are removed ${ }^{129}$. Interestingly, SYG-1 appears to prevent synapse elimination by preventing the assembly of a SCF ubiquitin ligase complex containing the SKP1 orthologue SKR1, Cullin, and the F-box protein SEL-10. Genetic data indicate that all three SCF complex components are required for proper synapse elimination in HSNs. The underlying mechanisms of SYG-1 dependent negative regulation of SCF activity in synapse elimination involves the cytoplasmic region of SYG-1 binding to SKR-1, which blocks the assembly of SKR-1 containing SCF complexes in HSNs. Thus, inactivation rather than activation of a specific ubiquitination pathway is crucial for synapse stability, which might well represent a general principle in synapse elimination processes during brain development.

The role of protein ubiquitination in synaptogenesis and synapse elimination has been studied extensively in invertebrates. However, the genes involved - and described above - may operate by different mechanisms in mammalian neurons. For instance, mice lacking Phr1, the murine rpm-1/Highwire orthologue, show perturbed axon formation ${ }^{128}$. Importantly, however, the expression level of DLK is not upregulated in this mutant, indicating that the function of mammalian Phr1 is different from that of invertebrate orthologues. In addition, recent studies identified novel E3 ligase dependent processes in mammalian synaptogenesis and synapse function. For example, the E3 ligases SCRAPPER ${ }^{130}$ and Ube3A ${ }^{131}$ regulate pre- and postsynapse formation and function by polyubiquitinating the active zone protein RIM1 $\alpha$ and the synaptic plasticity regulator Arc/Arg3.1, respectively, thus causing their degradation. The latter studies indicate that it will be of eminent importance to invest further resources into the analysis of mutant mouse lines with deletions of synapse-enriched E3 ligases in order to decipher the role of specific ubiquitination in synaptogenesis of mammalian neuron. 


\section{Conclusions and perspectives}

True to its name, protein ubiquitination ultimately affects most cellular process in eukaryotic cells, simply because many proteins are turned over under physiological conditions in a ubiquitination-dependent manner. In view of this 'catholic' importance of ubiquitination in cell biology, the recent progress in our understanding of the role of ubiquitination in nerve cell development is barely scratching the surface.

Nevertheless, a first conclusion that can be drawn from the present review on the role of protein ubiquitination in neuronal development - and which is somewhat trivial in view of findings in other areas of cell biology - is that protein ubiquitination is not just a refuse disposal service to developing nerve cells that targets proteins for UPS dependent degradation and that operates in the background of more sophisticated molecular processes. On the contrary, the examples discussed in the present review already show that protein ubiquitination is a regulatory principle whose complexity and importance are comparable to other key signaling processes in eukaryotic cells that are based on posttranslational protein modifications, such as phosphorylation. It operates by and is subject to the same cybernetic control mechanisms (e.g. feed-back and feed-forward control) and is used by cells for the same purposes (e.g. in cell surface signaling, intracellular signaling cascades, and transcriptional control), mostly in combination with other regulatory mechanisms. Even individual enzymes of ubiquitination pathways are regulated by similar biochemical mechanisms as protein kinases, such as allosteric regulation by second messengers ${ }^{132-134}$ or posttranslational modifications 135 .

The omnipresent nature of protein ubiquitination is probably the most profound obstacle for studies on its role in distinct cell biological processes - every cell contains thousands of different ubiquitination substrates. A promising entry-point for studies on defined cell biological roles of protein ubiquitination may be the E3 ubiquitin ligases, which are the key specificity determinants of ubiquitination. Higher eukaryotic genomes contain some 600 E3 ubiquitin ligase genes ${ }^{9}$, and the genetic and biochemical studies discussed in the present review demonstrate that analyses of the function of individual E3 ligases can yield direct and detailed insights into ubiquitination-controlled molecular processes, including relevant substrates and upstream or downstream signaling pathways. It is likely, that a systematic functional analysis of E3 ubiquitin ligases in developing neurons at the genetic and biochemical levels (e.g. by using cell-type specific or inducible mutants) will provide further insights into the role of ubiquitination in nerve cell development and the substrates involved. In this regard, the studies described in the present review can serve as conceptual project templates, and their complementation by comparative proteomic and biochemical approaches would be extremely helpful in the systematic identification of corresponding E3 ligase substrates.

Most E3 ligases interact with multiple substrate proteins through tandem target-recognition domains (e.g. WW domains of HECT type ligases) or by switching adaptor proteins (e.g. SCF complexes or APC). In this manner, a single E3 ligase can simultaneously regulate a large number of cytoplasmic or transmembrane proteins. The regulation of a subset of substrates must be coordinated to balance parallel regulatory pathways in neuronal differentiation or development. For example, Nedd4-1 ubiquitinates and thereby regulates Rap2 during dendrite development ${ }^{97}$ and may, at the same time, control axon branching by ubiquitinating PTEN ${ }^{98}$. It is obvious that such regulatory pathways must be properly adjusted in order to achieve proper neuronal network formation. Indeed, the selection of substrates depends on the given cell type and subcellular compartment, so that individual E3 ligases function effectively in cells abundant with the given ligase and in the subcellular compartments in which the ligase is enriched. In addition, intercalating signaling mechanisms such as phosphorylation can regulate E3 ligases in defined subcellular compartments to alter their activity or substrate preferences, as is the case for Smurf1 ${ }^{103}$. Thus, future work should not only examine the expression profiles of E3 ligases but also the upstream pathways by which they are localized and regulated.

The large superfamily of deubiquitinating enzymes represents a second important and promising entry point for the analysis of ubiquitination in neuronal development. The human genome encodes almost 100 such enzymes ${ }^{136}$, and multiple genetic and cell biological studies have demonstrated the involvement of specific deubiquitination processes in many regulatory ubiquitination pathways and signaling networks. Once a proteasome recognizes a polyubiquitin chain, the latter is removed from its substrate by deubiquitinating enzymes. Apart from this very well studied deubiquitination system, many deubiquitinating enzymes operate in a substrate specific manner or recognize only particular polyubiquitin chain types. The chain type specificity of certain deubiquitination enzymes adds an additional level of complexity to the protein ubiquitination system, which distinguishes it from protein phosphorylation where the balance between 
kinases and phosphatases alone is the main determinant of substrate phosphorylation. Yet another level of complexity of the ubiquitination system is contributed by the fact that some deubiquitinating enzymes specifically hydrolyse unanchored free polyubiquitin chains, which were recently shown to be physiologically relevant signaling components, e.g. in the control of certain transcription factors ${ }^{137}$.

In summary, multiple recent studies have shown that substrate specific protein ubiquitination plays a key role in brain development. However, the neurodevelopmental aspects of some of the most interesting and unique features of ubiquitination - e.g. the coordination of ubiquitination of multiple substrates by E3 ligases, the regulation and function of deubiquitinating enzymes, or the physiological role of unanchored polyubiquitin chains - have not been studied in much detail yet, especially not in vivo. It is very likely, that studies on these unique features of ubiquitination, along with more conventional analyses of ubiquitination cascades, e.g. by perturbing E3 ligase function, will provide key insights into all aspects of brain development and function. 


\section{References}

1. Lange, W. Cell number and cell density in the cerebellar cortex of man and some other mammals. Cell Tissue Res 157, 115-24 (1975).

2. Shariff, G.A. Cell counts in the primate cerebral cortex. J Comp Neurol 98, 381-400 (1953).

3. Barnes, A.P. \& Polleux, F. Establishment of axon-dendrite polarity in developing neurons. Annu Rev Neurosci 32, 347-81 (2009).

4. Parrish, J.Z., Emoto, K., Kim, M.D. \& Jan, Y.N. Mechanisms that regulate establishment, maintenance, and remodeling of dendritic fields. Annu Rev Neurosci 30, 399-423 (2007).

5. Spruston, N. Pyramidal neurons: dendritic structure and synaptic integration. Nat Rev Neurosci 9, 206-21 (2008).

6. O'Donnell, M., Chance, R.K. \& Bashaw, G.J. Axon growth and guidance: receptor regulation and signal transduction. Annu Rev Neurosci 32, 383-412 (2009).

7. Sanes, J.R. \& Yamagata, M. Many paths to synaptic specificity. Annu Rev Cell Dev Biol 25, 161-95 (2009).

8. Marin, O., Valiente, M., Ge, X. \& Tsai, L.H. Guiding neuronal cell migrations. Cold Spring Harb Perspect Biol 2, a001834 (2010).

9. Li, W. et al. Genome-wide and functional annotation of human E3 ubiquitin ligases identifies MULAN, a mitochondrial E3 that regulates the organelle's dynamics and signaling. PLoS One 3, e1487 (2008).

10. $\mathrm{Xu}, \mathrm{P}$. et al. Quantitative proteomics reveals the function of unconventional ubiquitin chains in proteasomal degradation. Cell 137, 133-45 (2009).

11. Kim, H.C. \& Huibregtse, J.M. Polyubiquitination by HECT E3s and the determinants of chain type specificity. Mol Cell Biol 29, 3307-18 (2009).

12. Hicke, L. Protein regulation by monoubiquitin. Nat Rev Mol Cell Biol 2, 195-201 (2001).

13. Xu, L., Lubkov, V., Taylor, L.J. \& Bar-Sagi, D. Feedback regulation of Ras signaling by Rabex-5mediated ubiquitination. Curr Biol 20, 1372-7 (2010).

14. Wilkinson, K.D. et al. The neuron-specific protein PGP 9.5 is a ubiquitin carboxyl-terminal hydrolase. Science 246, 670-3 (1989).

15. Kent, C. \& Clarke, P.J. The immunolocalisation of the neuroendocrine specific protein PGP9.5 during neurogenesis in the rat. Brain Res Dev Brain Res 58, 147-50 (1991).

16. Lowe, J. et al. A filamentous inclusion body within anterior horn neurones in motor neurone disease defined by immunocytochemical localisation of ubiquitin. Neurosci Lett 94, 203-10 (1988).

17. Lennox, G., Lowe, J., Morrell, K., Landon, M. \& Mayer, R.J. Ubiquitin is a component of neurofibrillary tangles in a variety of neurodegenerative diseases. Neurosci Lett 94, 211-7 (1988).

18. Tai, H.C. \& Schuman, E.M. Ubiquitin, the proteasome and protein degradation in neuronal function and dysfunction. Nat Rev Neurosci 9, 826-38 (2008).

19. Qian, X. et al. Timing of CNS cell generation: a programmed sequence of neuron and glial cell production from isolated murine cortical stem cells. Neuron 28, 69-80 (2000).

20. Gerhart, J. 1998 Warkany lecture: signaling pathways in development. Teratology 60, 226-39 (1999).

21. Yun, S.J. et al. Transcriptional regulatory networks associated with self-renewal and differentiation of neural stem cells. J Cell Physiol (2010).

22. Inestrosa, N.C. \& Arenas, E. Emerging roles of Wnts in the adult nervous system. Nat Rev Neurosci 11, 77-86 (2010). 
23. Kikuchi, A. Regulation of beta-catenin signaling in the Wnt pathway. Biochem Biophys Res Commun 268, 243-8 (2000).

24. Yoon, K. \& Gaiano, N. Notch signaling in the mammalian central nervous system: insights from mouse mutants. Nat Neurosci 8, 709-15 (2005).

25. Schier, A.F. et al. Mutations affecting the development of the embryonic zebrafish brain. Development 123, 165-78 (1996).

26. Jiang, Y.J. et al. Mutations affecting neurogenesis and brain morphology in the zebrafish, Danio rerio. Development 123, 205-16 (1996).

27. Itoh, M. et al. Mind bomb is a ubiquitin ligase that is essential for efficient activation of Notch signaling by Delta. Dev Cell 4, 67-82 (2003).

This study shows that the $\mathbf{E} 3$ ubiquitin ligase mind bomb ubiquitinates Delta and thereby regulates Notch signaling.

28. Yoon, K.J. et al. Mind bomb 1-expressing intermediate progenitors generate notch signaling to maintain radial glial cells. Neuron 58, 519-31 (2008).

Using conditional Mind bomb1 knock-out mice, the authors of this study provide important evidence for the regulation of Delta by Mind bomb1 during neurogenesis.

29. Koo, B.K. et al. Mind bomb 1 is essential for generating functional Notch ligands to activate Notch. Development 132, 3459-70 (2005).

30. Wang, W. \& Struhl, G. Distinct roles for Mind bomb, Neuralized and Epsin in mediating DSL endocytosis and signaling in Drosophila. Development 132, 2883-94 (2005).

31. Yamamoto, M. et al. Mib-Jag1-Notch signalling regulates patterning and structural roles of the notochord by controlling cell-fate decisions. Development 137, 2527-37 (2010).

32. Konno, D. et al. Neuroepithelial progenitors undergo LGN-dependent planar divisions to maintain self-renewability during mammalian neurogenesis. Nat Cell Biol 10, 93-101 (2008).

33. Ossipova, O., Ezan, J. \& Sokol, S.Y. PAR-1 phosphorylates Mind bomb to promote vertebrate neurogenesis. Dev Cell 17, 222-33 (2009).

34. Knoepfler, P.S., Cheng, P.F. \& Eisenman, R.N. N-myc is essential during neurogenesis for the rapid expansion of progenitor cell populations and the inhibition of neuronal differentiation. Genes Dev 16, 2699-712 (2002).

35. Zhao, X. et al. The HECT-domain ubiquitin ligase Huwel controls neural differentiation and proliferation by destabilizing the N-Myc oncoprotein. Nat Cell Biol 10, 643-53 (2008).

36. Zhao, X. et al. The N-Myc-DLL3 cascade is suppressed by the ubiquitin ligase Huwe1 to inhibit proliferation and promote neurogenesis in the developing brain. Dev Cell 17, 210-21 (2009).

37. Marthiens, V. \& ffrench-Constant, C. Adherens junction domains are split by asymmetric division of embryonic neural stem cells. EMBO Rep 10, 515-20 (2009).

38. Marquardt, T. et al. Pax6 is required for the multipotent state of retinal progenitor cells. Cell 105, 4355 (2001).

39. Hebert, J.M. \& Fishell, G. The genetics of early telencephalon patterning: some assembly required. Nat Rev Neurosci 9, 678-85 (2008).

40. St-Onge, L., Sosa-Pineda, B., Chowdhury, K., Mansouri, A. \& Gruss, P. Pax6 is required for differentiation of glucagon-producing alpha-cells in mouse pancreas. Nature 387, 406-9 (1997).

41. Englund, C. et al. Pax6, Tbr2, and Tbr1 are expressed sequentially by radial glia, intermediate progenitor cells, and postmitotic neurons in developing neocortex. J Neurosci 25, 247-51 (2005).

42. Scardigli, R., Baumer, N., Gruss, P., Guillemot, F. \& Le Roux, I. Direct and concentrationdependent regulation of the proneural gene Neurogenin2 by Pax6. Development 130, 3269-81 (2003). 
43. Tuoc, T.C. \& Stoykova, A. Trim11 modulates the function of neurogenic transcription factor Pax6 through ubiquitin-proteosome system. Genes Dev 22, 1972-86 (2008).

44. Berger, J. et al. Conditional activation of Pax6 in the developing cortex of transgenic mice causes progenitor apoptosis. Development 134, 1311-22 (2007).

45. Sobieszczuk, D.F., Poliakov, A., Xu, Q. \& Wilkinson, D.G. A feedback loop mediated by degradation of an inhibitor is required to initiate neuronal differentiation. Genes Dev 24, 206-18 (2010).

46. Kageyama, R., Ohtsuka, T., Shimojo, H. \& Imayoshi, I. Dynamic regulation of Notch signaling in neural progenitor cells. Curr Opin Cell Biol 21, 733-40 (2009).

47. Shimojo, H., Ohtsuka, T. \& Kageyama, R. Oscillations in notch signaling regulate maintenance of neural progenitors. Neuron 58, 52-64 (2008).

48. Hirata, H. et al. Oscillatory expression of the bHLH factor Hes1 regulated by a negative feedback loop. Science 298, 840-3 (2002).

49. Hirabayashi, Y. et al. Polycomb limits the neurogenic competence of neural precursor cells to promote astrogenic fate transition. Neuron 63, 600-13 (2009).

This study describes the role of the PcG components PRC1 and PRC2 in fate decision of neural progenitor cells and shows that the function of the ubiquitin ligase Ring1B in the PRC2 complex is essential for this process.

50. Fan, G. et al. DNA methylation controls the timing of astrogliogenesis through regulation of JAKSTAT signaling. Development 132, 3345-56 (2005).

51. $\mathrm{Wu}, \mathrm{H}$. et al. Dnmt3a-dependent nonpromoter DNA methylation facilitates transcription of neurogenic genes. Science 329, 444-8 (2010).

52. Hatada, I. et al. Astrocyte-specific genes are generally demethylated in neural precursor cells prior to astrocytic differentiation. PLoS One 3, e3189 (2008).

53. Namihira, M., Nakashima, K. \& Taga, T. Developmental stage dependent regulation of DNA methylation and chromatin modification in a immature astrocyte specific gene promoter. FEBS Lett 572, 184-8 (2004).

54. Takizawa, $\mathrm{T}$. et al. DNA methylation is a critical cell-intrinsic determinant of astrocyte differentiation in the fetal brain. Dev Cell 1, 749-58 (2001).

55. Cao, R. et al. Role of histone H3 lysine 27 methylation in Polycomb-group silencing. Science 298, 1039-43 (2002).

56. Wang, H. et al. Role of histone H2A ubiquitination in Polycomb silencing. Nature 431, 873-8 (2004).

57. Cao, R., Tsukada, Y. \& Zhang, Y. Role of Bmi-1 and Ring1A in H2A ubiquitylation and Hox gene silencing. Mol Cell 20, 845-54 (2005).

58. Mohd-Sarip, A., Venturini, F., Chalkley, G.E. \& Verrijzer, C.P. Pleiohomeotic can link polycomb to DNA and mediate transcriptional repression. Mol Cell Biol 22, 7473-83 (2002).

59. Mohd-Sarip, A., Cleard, F., Mishra, R.K., Karch, F. \& Verrijzer, C.P. Synergistic recognition of an epigenetic DNA element by Pleiohomeotic and a Polycomb core complex. Genes Dev 19, 1755-60 (2005).

60. Mohd-Sarip, A. et al. Architecture of a polycomb nucleoprotein complex. Mol Cell 24, 91-100 (2006).

61. Chong, J.A. et al. REST: a mammalian silencer protein that restricts sodium channel gene expression to neurons. Cell 80, 949-57 (1995).

62. Schoenherr, C.J. \& Anderson, D.J. The neuron-restrictive silencer factor (NRSF): a coordinate repressor of multiple neuron-specific genes. Science 267, 1360-3 (1995). 
63. Lee, M.G., Wynder, C., Cooch, N. \& Shiekhattar, R. An essential role for CoREST in nucleosomal histone 3 lysine 4 demethylation. Nature 437, 432-5 (2005).

64. Roopra, A., Qazi, R., Schoenike, B., Daley, T.J. \& Morrison, J.F. Localized domains of G9amediated histone methylation are required for silencing of neuronal genes. Mol Cell 14, 727-38 (2004).

65. Lunyak, V.V. et al. Corepressor-dependent silencing of chromosomal regions encoding neuronal genes. Science 298, 1747-52 (2002).

66. Ballas, N., Grunseich, C., Lu, D.D., Speh, J.C. \& Mandel, G. REST and its corepressors mediate plasticity of neuronal gene chromatin throughout neurogenesis. Cell 121, 645-57 (2005).

67. Westbrook, T.F. et al. SCFbeta-TRCP controls oncogenic transformation and neural differentiation through REST degradation. Nature 452, 370-4 (2008).

In this paper, the authors show that the ubiquitination and degradation of REST by SCF-b-TrCP is required for the differentiation of neural progenitor cells to neurons.

68. Kohyama, J. et al. BMP-induced REST regulates the establishment and maintenance of astrocytic identity. J Cell Biol 189, 159-70 (2010).

69. Hamburgh, M. Analysis of the Postnatal Developmental Effects of "Reeler," a Neurological Mutation in Mice. a Study in Developmental Genetics. Dev Biol 19, 165-85 (1963).

70. Senzaki, K., Ogawa, M. \& Yagi, T. Proteins of the CNR family are multiple receptors for Reelin. Cell 99, 635-47 (1999).

71. Hiesberger, T. et al. Direct binding of Reelin to VLDL receptor and ApoE receptor 2 induces tyrosine phosphorylation of disabled-1 and modulates tau phosphorylation. Neuron 24, 481-9 (1999).

72. D'Arcangelo, G. et al. Reelin is a ligand for lipoprotein receptors. Neuron 24, 471-9 (1999).

73. Trommsdorff, M. et al. Reeler/Disabled-like disruption of neuronal migration in knockout mice lacking the VLDL receptor and ApoE receptor 2. Cell 97, 689-701 (1999).

74. Keshvara, L., Benhayon, D., Magdaleno, S. \& Curran, T. Identification of reelin-induced sites of tyrosyl phosphorylation on disabled 1. J Biol Chem 276, 16008-14 (2001).

75. Sheldon, M. et al. Scrambler and yotari disrupt the disabled gene and produce a reeler-like phenotype in mice. Nature 389, 730-3 (1997).

76. Ware, M.L. et al. Aberrant splicing of a mouse disabled homolog, mdab1, in the scrambler mouse. Neuron 19, 239-49 (1997).

77. Arnaud, L., Ballif, B.A. \& Cooper, J.A. Regulation of protein tyrosine kinase signaling by substrate degradation during brain development. Mol Cell Biol 23, 9293-302 (2003).

78. Feng, L., Allen, N.S., Simo, S. \& Cooper, J.A. Cullin 5 regulates Dab1 protein levels and neuron positioning during cortical development. Genes Dev 21, 2717-30 (2007).

79. Simo, S., Jossin, Y. \& Cooper, J.A. Cullin 5 regulates cortical layering by modulating the speed and duration of Dab1-dependent neuronal migration. J Neurosci 30, 5668-76 (2010).

80. Tissir, F. \& Goffinet, A.M. Reelin and brain development. Nat Rev Neurosci 4, 496-505 (2003).

81. Campbell, D.S. \& Holt, C.E. Chemotropic responses of retinal growth cones mediated by rapid local protein synthesis and degradation. Neuron 32, 1013-26 (2001).

82. Kim, T.H. et al. Netrin induces down-regulation of its receptor, Deleted in Colorectal Cancer, through the ubiquitin-proteasome pathway in the embryonic cortical neuron. $J$ Neurochem 95, 1-8 (2005).

83. $\mathrm{Hu}, \mathrm{G}$. et al. Mammalian homologs of seven in absentia regulate DCC via the ubiquitin-proteasome pathway. Genes Dev 11, 2701-14 (1997).

84. Yuasa-Kawada, J., Kinoshita-Kawada, M., Wu, G., Rao, Y. \& Wu, J.Y. Midline crossing and Slit responsiveness of commissural axons require USP33. Nat Neurosci 12, 1087-9 (2009). 
This study identifies the deubiquitinating enzyme USP33 as a binding partner of Robo1 and shows that USP33 is critical for midline crossing of axons.

85. Piper, M. et al. Signaling mechanisms underlying Slit2-induced collapse of Xenopus retinal growth cones. Neuron 49, 215-28 (2006).

86. Tojima, T., Hines, J.H., Henley, J.R. \& Kamiguchi, H. Second messengers and membrane trafficking direct and organize growth cone steering. Nat Rev Neurosci 12, 191-203 (2011).

87. Takai, Y., Sasaki, T. \& Matozaki, T. Small GTP-binding proteins. Physiol Rev 81, 153-208 (2001).

88. Arimura, N. \& Kaibuchi, K. Neuronal polarity: from extracellular signals to intracellular mechanisms. Nat Rev Neurosci 8, 194-205 (2007).

89. da Silva, J.S. \& Dotti, C.G. Breaking the neuronal sphere: regulation of the actin cytoskeleton in neuritogenesis. Nat Rev Neurosci 3, 694-704 (2002).

90. Garvalov, B.K. et al. Cdc42 regulates cofilin during the establishment of neuronal polarity. $J$ Neurosci 27, 13117-29 (2007).

91. Schwamborn, J.C. \& Puschel, A.W. The sequential activity of the GTPases Rap1B and Cdc42 determines neuronal polarity. Nat Neurosci 7, 923-9 (2004).

92. Fu, Z. et al. Differential roles of Rap1 and Rap2 small GTPases in neurite retraction and synapse elimination in hippocampal spiny neurons. $J$ Neurochem 100, 118-31 (2007).

93. Bryan, B. et al. Ubiquitination of RhoA by Smurf1 promotes neurite outgrowth. FEBS Lett 579, 1015-9 (2005).

94. Wang, H.R. et al. Regulation of cell polarity and protrusion formation by targeting RhoA for degradation. Science 302, 1775-9 (2003).

95. Schwamborn, J.C., Muller, M., Becker, A.H. \& Puschel, A.W. Ubiquitination of the GTPase Rap1B by the ubiquitin ligase Smurf2 is required for the establishment of neuronal polarity. Embo $J$ 26, 1410-22 (2007).

This paper provides the first evidence that the expression level of a small GTPase is regulated by polyubiquitination and plays a key role in neuronal development.

96. Jura, N., Scotto-Lavino, E., Sobczyk, A. \& Bar-Sagi, D. Differential modification of Ras proteins by ubiquitination. Mol Cell 21, 679-87 (2006).

97. Kawabe, H. et al. Regulation of Rap2A by the ubiquitin ligase Nedd4-1 controls neurite development. Neuron 65, 358-72 (2010).

This study shows that the HECT type E3 ligase Nedd4-1 controls dendrite growth by monoubiquitination and inhibition of Rap2 and consequent inhibition of the kinase TNIK.

98. Drinjakovic, J. et al. E3 ligase Nedd4 promotes axon branching by downregulating PTEN. Neuron 65, 341-57 (2010).

99. Trotman, L.C. et al. Ubiquitination regulates PTEN nuclear import and tumor suppression. Cell 128, 141-56 (2007).

100. Wang, X. et al. NEDD4-1 is a proto-oncogenic ubiquitin ligase for PTEN. Cell 128, 129-39 (2007).

101. Fouladkou, F. et al. The ubiquitin ligase Nedd4-1 is dispensable for the regulation of PTEN stability and localization. Proc Natl Acad Sci U S A 105, 8585-90 (2008).

102. Rotin, D. \& Kumar, S. Physiological functions of the HECT family of ubiquitin ligases. Nat Rev Mol Cell Biol 10, 398-409 (2009).

103. Cheng, P.L., Lu, H., Shelly, M., Gao, H. \& Poo, M.M. Phosphorylation of E3 ligase Smurf1 switches its substrate preference in support of axon development. Neuron 69, 231-43 (2011).

This paper shows that phosphorylation of Smurf1 switches its substrate preference from Par6 to RhoA in axons. 
104. Thornton, B.R. \& Toczyski, D.P. Precise destruction: an emerging picture of the APC. Genes Dev 20, 3069-78 (2006).

105. Konishi, Y., Stegmuller, J., Matsuda, T., Bonni, S. \& Bonni, A. Cdh1-APC controls axonal growth and patterning in the mammalian brain. Science 303, 1026-30 (2004).

This is the first paper to demonstrate a key function of APC in postmitotic neurons.

106. Stegmuller, J. et al. Cell-intrinsic regulation of axonal morphogenesis by the Cdh1-APC target SnoN. Neuron 50, 389-400 (2006).

107. Kim, A.H. et al. A centrosomal Cdc20-APC pathway controls dendrite morphogenesis in postmitotic neurons. Cell 136, 322-36 (2009).

108. Huynh, M.A., Stegmuller, J., Litterman, N. \& Bonni, A. Regulation of Cdh1-APC function in axon growth by Cdh1 phosphorylation. J Neurosci 29, 4322-7 (2009).

109. Zhou, Y., Ching, Y.P., Chun, A.C. \& Jin, D.Y. Nuclear localization of the cell cycle regulator CDH1 and its regulation by phosphorylation. J Biol Chem 278, 12530-6 (2003).

110. Yang, Y. et al. A Cdc20-APC ubiquitin signaling pathway regulates presynaptic differentiation. Science 326, 575-8 (2009).

111. Margeta, M.A. \& Shen, K. Molecular mechanisms of synaptic specificity. Mol Cell Neurosci 43, 261-7 (2010).

112. Huttenlocher, P.R., de Courten, C., Garey, L.J. \& Van der Loos, H. Synaptogenesis in human visual cortex--evidence for synapse elimination during normal development. Neurosci Lett 33, 247-52 (1982).

113. Zecevic, N. \& Rakic, P. Synaptogenesis in monkey somatosensory cortex. Cereb Cortex 1, 510-23 (1991).

114. DiAntonio, A. et al. Ubiquitination-dependent mechanisms regulate synaptic growth and function. Nature 412, 449-52 (2001).

This paper is of particular importance as it demonstrates that protein ubiquitination is crucial for synaptogenesis.

115. Wan, H.I. et al. Highwire regulates synaptic growth in Drosophila. Neuron 26, 313-29 (2000).

116. Zhen, M., Huang, X., Bamber, B. \& Jin, Y. Regulation of presynaptic terminal organization by C. elegans RPM-1, a putative guanine nucleotide exchanger with a RING-H2 finger domain. Neuron 26, 331-43 (2000).

117. Schaefer, A.M., Hadwiger, G.D. \& Nonet, M.L. rpm-1, a conserved neuronal gene that regulates targeting and synaptogenesis in C. elegans. Neuron 26, 345-56 (2000).

118. McCabe, B.D. et al. Highwire regulates presynaptic BMP signaling essential for synaptic growth. Neuron 41, 891-905 (2004).

119. Nakata, K. et al. Regulation of a DLK-1 and p38 MAP kinase pathway by the ubiquitin ligase RPM1 is required for presynaptic development. Cell 120, 407-20 (2005).

This study extends the characterization of the PHR ligase and identifies the DLK-1/MKK-4/PMK-3 cascade as a downstream effector pathway of PHR.

120. Li, H., Kulkarni, G. \& Wadsworth, W.G. RPM-1, a Caenorhabditis elegans protein that functions in presynaptic differentiation, negatively regulates axon outgrowth by controlling SAX-3/robo and UNC-5/UNC5 activity. J Neurosci 28, 3595-603 (2008).

121. Grill, B. et al. C. elegans RPM-1 regulates axon termination and synaptogenesis through the Rab GEF GLO-4 and the Rab GTPase GLO-1. Neuron 55, 587-601 (2007).

122. Collins, C.A., Wairkar, Y.P., Johnson, S.L. \& DiAntonio, A. Highwire restrains synaptic growth by attenuating a MAP kinase signal. Neuron 51, 57-69 (2006). 
123. Yan, D., Wu, Z., Chisholm, A.D. \& Jin, Y. The DLK-1 kinase promotes mRNA stability and local translation in C. elegans synapses and axon regeneration. Cell 138, 1005-18 (2009).

124. Liao, E.H., Hung, W., Abrams, B. \& Zhen, M. An SCF-like ubiquitin ligase complex that controls presynaptic differentiation. Nature 430, 345-50 (2004).

125. Wu, C., Daniels, R.W. \& DiAntonio, A. DFsn collaborates with Highwire to down-regulate the Wallenda/DLK kinase and restrain synaptic terminal growth. Neural Dev 2, 16 (2007).

126. Burgess, R.W. et al. Evidence for a conserved function in synapse formation reveals Phr1 as a candidate gene for respiratory failure in newborn mice. Mol Cell Biol 24, 1096-105 (2004).

127. Tada, H. et al. Fbxo45, a novel ubiquitin ligase, regulates synaptic activity. J Biol Chem 285, 3840-9 (2010).

128. Bloom, A.J., Miller, B.R., Sanes, J.R. \& DiAntonio, A. The requirement for Phr1 in CNS axon tract formation reveals the corticostriatal boundary as a choice point for cortical axons. Genes Dev 21, 2593-606 (2007).

129. Ding, M., Chao, D., Wang, G. \& Shen, K. Spatial regulation of an E3 ubiquitin ligase directs selective synapse elimination. Science 317, 947-51 (2007).

This study describes the mechanism by which the regulation of a specific SCF complex controls synapse elimination.

130. Yao, I. et al. SCRAPPER-dependent ubiquitination of active zone protein RIM1 regulates synaptic vesicle release. Cell 130, 943-57 (2007).

131. Greer, P.L. et al. The Angelman Syndrome protein Ube3A regulates synapse development by ubiquitinating arc. Cell 140, 704-16 (2010).

132. Wiesner, S. et al. Autoinhibition of the HECT-type ubiquitin ligase Smurf2 through its C2 domain. Cell 130, 651-62 (2007).

133. Wang, J. et al. Calcium activates Nedd4 E3 ubiquitin ligases by releasing the C2 domain-mediated auto-inhibition. J Biol Chem 285, 12279-88 (2010).

134. Plant, P.J., Yeger, H., Staub, O., Howard, P. \& Rotin, D. The C2 domain of the ubiquitin protein ligase Nedd4 mediates Ca2+-dependent plasma membrane localization. J Biol Chem 272, 32329-36 (1997).

135. Saha, A. \& Deshaies, R.J. Multimodal activation of the ubiquitin ligase SCF by Nedd8 conjugation. Mol Cell 32, 21-31 (2008).

136. Reyes-Turcu, F.E., Ventii, K.H. \& Wilkinson, K.D. Regulation and cellular roles of ubiquitinspecific deubiquitinating enzymes. Annu Rev Biochem 78, 363-97 (2009).

137. Zeng, W. et al. Reconstitution of the RIG-I pathway reveals a signaling role of unanchored polyubiquitin chains in innate immunity. Cell 141, 315-30 (2010). 


\section{Acknowledgements}

We thank Dr. Judith Stegmüller for helpful discussions and comments on this manuscript. Work in the authors' laboratories was supported by grants from the Max Planck Society (to N.B.), the German Research Foundation (SFB271/B11 to N.B.), the European Commission (EUSynapse, EUROSPIN, SynSys to N.B), and the Fritz Thyssen Foundation (to H.K.). 